Pacific

Journal of

Mathematics

ASYMPTOTIC ESTIMATION FOR A $p$-GINZBURG-LANDAU TYPE MINIMIZER IN HIGHER DIMENSIONS

YUTIAN LEI 


\title{
ASYMPTOTIC ESTIMATION FOR A $p$-GINZBURG-LANDAU TYPE MINIMIZER IN HIGHER DIMENSIONS
}

\author{
YUTIAN LEI
}

\begin{abstract}
This paper is concerned with the asymptotic behavior of the minimizers $u_{\varepsilon}$ of a $p$-Ginzburg-Landau type functional when $\varepsilon \rightarrow 0$. First the author discusses the location of zeros of $u_{\varepsilon}$ qualitatively. Then the $W^{1, p}$ estimation of $u_{\varepsilon}$ is set up. Finally, the author proves the $C^{1, \alpha}$ convergence of $u_{\varepsilon}$.
\end{abstract}

\section{Introduction}

Let $B_{r}=\left\{x \in R^{n} ;|x|<r\right\}$, where $n \geq 2$. Denote by $u_{\varepsilon}$ the minimizer of the functional

$$
E_{\varepsilon}(u)=\frac{1}{2} \int_{B_{1}}|\nabla u|^{2}+\frac{1}{4 \varepsilon^{2}} \int_{B_{1} \backslash B_{\varrho}}\left(1-|u|^{2}\right)^{2}+\frac{1}{2 \varepsilon^{2}} \int_{B_{\varrho}}|u|^{2}
$$

in the function space $H_{g}^{1}\left(B_{1}, R^{n}\right)$, where the mapping $g: \partial B_{1} \rightarrow S^{n-1}$ is smooth and satisfies $\operatorname{deg}\left(g, \partial B_{1}\right)=d \neq 0$. The functional $E_{\varepsilon}(u)$ with $n=2$ is related to the Ginzburg-Landau model of superconductivity with normal impurity inclusion such as superconducting-normal junctions [Chapman et al. 1995]. To represent the domains occupied by the superconducting materials and the normal conducting materials, we use $B_{1} \backslash \bar{B}_{\varrho}$ and $B_{\varrho}$, respectively. The minimizer $u_{\varepsilon}$ is the order parameter. In the physics literature, $u_{\varepsilon}$ is called a Higgs field. The parameter $\varepsilon$, which has the dimension of length, depends on the material and its temperature. When the temperature is not too close to the critical temperature, $\varepsilon$ is extremely small. The zeros of $u_{\varepsilon}$ exist in $B_{1}$ since $d \neq 0$. They are known as the GinzburgLandau vortices which are of significance in the theory of superconductivity [Du et al. 1992; Tinkham 1975]. The asymptotic behavior of the minimizer $u_{\varepsilon}$ was studied when both $\varepsilon$ and $\varrho$ converge to 0 , and the vortex-pinning effect was discussed [Ding et al. 1998].

MSC2000: 35J25, 35J70, 49K20.

Keywords: regularized minimizer, asymptotic behavior, $p$-Ginzburg-Landau functional, location of zeros.

The author is grateful to the referees for their helpful comments. He also thanks Prof. Yujun Dong for correcting some inappropriate English expressions. The research was supported in part by NSF (10571087) of China, and Science Foundation of Jiangsu Province Education Department (04KJB110062). 
Now, we consider the minimizer of

$$
E_{\varepsilon}\left(u, B_{1}\right)=\frac{1}{p} \int_{B_{1}}|\nabla u|^{p}+\frac{1}{4 \varepsilon^{p}} \int_{B_{1} \backslash B_{\varrho}}\left(1-|u|^{2}\right)^{2}+\frac{1}{2 \varepsilon^{p}} \int_{B_{\varrho}}|u|^{2}
$$

with $p>1$ and $p \neq n$ in the class $W=W_{g}^{1, p}\left(B_{1}, R^{n}\right)$. By means of the calculus of variations, we can see the existence of minimizers $u_{\varepsilon}$. As in [Ding et al. 1998], we are concerned with the asymptotic behavior of $u_{\varepsilon}$ when $\varepsilon$ and $\varrho$ tend to 0 . In this paper, we discuss the problem in the following cases:

Case I: $\varrho \leq \varepsilon$ or $\quad \varrho=O(\varepsilon)$ as $\varepsilon \rightarrow 0$;

Case II: $\varrho>\varepsilon$ and $\lim _{\varrho \rightarrow 0} \varepsilon / \varrho=0$.

In Case I, by the same argument proving Theorem III.1 in [Bethuel et al. 1994], we can easily see that

$$
E_{\varepsilon}\left(u_{\varepsilon}, B_{1}\right) \leq C\left(1+\varepsilon^{n-p}\right) .
$$

However, in Case II, the proof of (1-1) seems to be difficult. In Section 2, we will establish the estimation for $E_{\varepsilon}\left(u_{\varepsilon}, B_{1} \backslash B_{\gamma_{\varrho}}\right)$ with $\gamma>1$; see Proposition 2.4. Based on these results, in Section 3 we describe the vortex-pinning effect, i.e., the location of the zeros of the minimizer.

Theorem 1.1. Assume $u_{\varepsilon}$ is a minimizer. Then there are finitely many points $a_{1}, a_{2}, \ldots, a_{N} \in \bar{B}_{1}$, such that for any $\eta \in(0,1 / 2)$, there is $h=h(\eta)>0$ which is independent of $\varepsilon, \varrho \in\left(0, \varepsilon_{0}\right)$ with $\varepsilon_{0}$ sufficiently small, satisfying the following properties:

In Case I,

$$
\left\{x \in B_{1} ;\left|u_{\varepsilon}(x)\right|<1-\eta\right\} \subset \bigcup_{i=1}^{N} B\left(a_{i}, h \varepsilon\right) \cup\left(B_{h \varepsilon} \cup B_{\varrho}\right) .
$$

If $h \varepsilon<\varrho \leq \varepsilon$, then

$$
\left\{x \in B_{\varrho} ;\left|u_{\varepsilon}(x)\right| \geq \eta\right\} \subset B_{h \varepsilon} \quad \text { and } \quad\left|u_{\varepsilon}(x)\right|<\eta, \forall x \in B_{\varrho} \backslash B_{h \varepsilon} .
$$

In Case II with $p>n$, there exists $\gamma>1$ such that

$$
\left\{x \in B_{1} ;\left|u_{\varepsilon}(x)\right|<1-\eta\right\} \subset \bigcup_{i=1}^{N} B\left(a_{i}, h \varepsilon\right) \cup B_{\gamma \varrho} .
$$

In Case II with $n-t<p<n$, for $t$ a constant in $(0, \min \{1 / 2,4 / n\})$, we have

$$
\left\{x \in B_{1} ;\left|u_{\varepsilon}(x)\right|<1-\eta\right\} \subset \bigcup_{i=1}^{N} B\left(a_{i}, h \varrho^{(n-1) / n} \varepsilon^{1 / n}\right) \cup B_{\gamma} .
$$

Remark 1. If the vortices (zeros of $\left|u_{\varepsilon}\right|$ ) concentrate in some region, we talk of the pinning effect. According to Theorem 1.1, the vortices converge to $\left\{0, a_{1}, \ldots, a_{N}\right\}$ when both $\varrho$ and $\varepsilon$ tend to zero. When $h \varepsilon<\varrho \leq \varepsilon$, we investigate a fixed point $x_{0} \in B_{h \varepsilon} \backslash\{0\}$ satisfying $\left|u_{\varepsilon}\left(x_{0}\right)\right| \geq \eta$. In this situation, the superconductive state 
at $x_{0}$ appears. Letting $\varepsilon$ go to zero, when $\varepsilon$ becomes so small that $x_{0} \in B_{\varrho} \backslash B_{h \varepsilon}$, the normal conductive state at $x_{0}$ may appear, since $\left|u_{\varepsilon}\left(x_{0}\right)\right|<\eta$. As $\varepsilon$ becomes extremely small, $x_{0} \in B_{1 / 2} \backslash B_{\varrho}$, so $\left|u_{\varepsilon}\left(x_{0}\right)\right| \geq 1-\eta$. Again the superconductive state at $x_{0}$ appears. This shows the conductive state is complicated and may change near the origin when $\varepsilon$ and $\varrho$ tend to zero.

Next, we will set up the uniform estimation of $\left\|u_{\varepsilon}\right\|_{W^{1, p}}$. When $p>n$, the idea in [Ding et al. 1998] (coming from [Bethuel et al. 1994]) is not valid, since the coefficients $C_{2}$ and $C_{3}$ of $\varepsilon^{n-p}$ in the upper bounds for $E_{\varepsilon}\left(u_{\varepsilon}, B_{1}\right)$ and $E_{\varepsilon}\left(u_{\varepsilon}, B_{1} \backslash B_{\gamma \varrho}\right)$, respectively, are not sufficiently accurate. (See Propositions 2.3 and 2.4.) The reason is that the conformal transformation of $\int\left|\nabla u_{\varepsilon}\right|^{p} d x$ is lost when $p \neq n$. Although $E_{\varepsilon}\left(u_{\varepsilon},\left[B\left(a_{i}, R\right) \backslash B\left(a_{i}, h \varepsilon\right)\right] \cup\left[B_{R} \backslash B_{\gamma_{\varrho}}\right]\right)$ can be bounded below by $C_{4}\left(\varepsilon^{n-p}-1\right)$, the constant $C_{4}$ may be smaller than $C_{2}$ and $C_{3}$. Thus, it is impossible to get the uniform estimation of $E_{\varepsilon}\left(u_{\varepsilon}, K\right)$ as we do in the case $p=n$ [Bethuel et al. 1994; Ding et al. 1998; Han and Li 1996; Hong 1996], where $K$ is an arbitrary compact subset of $B_{1} \backslash\left\{0, a_{1}, a_{2}, \ldots, a_{N}\right\}$. In Section 4, we establish the uniform estimation by means of induction. However in the proof, there are few results linking the degrees of the zeros of $u_{\varepsilon}$ and the singularities of the p-harmonic maps. Hence, the relation between $d$ and $N$ is still open.

Theorem 1.2. Assume $u_{\varepsilon}$ is a minimizer. Then $\left|u_{\varepsilon}\right| \leq 1$ a.e. on $\bar{B}_{1}$. In addition, in Case I with $p \in(1, n)$, there exists a constant $C>0$ which is independent of $\varepsilon, \varrho \in\left(0, \varepsilon_{0}\right)$, such that

$$
E_{\varepsilon}\left(u_{\varepsilon}, B_{1}\right) \leq C \text {. }
$$

In Case I with $p>n$ or in Case II, for any compact $K \subset B_{1} \backslash\left\{0, a_{1}, a_{2}, \ldots, a_{N}\right\}$, there exists $C=C(K)>0$ such that

$$
E_{\varepsilon}\left(u_{\varepsilon}, K\right) \leq C
$$

Remark 2. Based on these results, we will set up the following convergences of the minimizer as $\varrho$ and $\varepsilon$ go to 0 :

(1) In Case I with $p \in(1, n)$, obviously, $E_{\varepsilon}\left(u_{\varepsilon}, B_{1}\right) \leq E_{\varepsilon}\left(u_{*}, B_{1}\right)$, where $u_{*}$ is a least map of the energy $\int_{B_{1}}|\nabla u|^{p} d x$ on $W_{g}^{1, p}\left(B_{1}, S^{n-1}\right)$. In addition, we have

$$
\lim _{\varepsilon \rightarrow 0} \frac{1}{\varepsilon^{p}} \int_{B_{\varrho}}\left|u_{*}\right|^{2} d x=C(\pi) \lim _{\varepsilon \rightarrow 0} \frac{\varrho^{n}}{\varepsilon^{p}}=0 .
$$

Thus, by the weak lower semicontinuity of $\int|\nabla u|^{p}$, there is a subsequence $u_{\varepsilon_{k}}$ of $u_{\varepsilon}$ such that as $\varepsilon \rightarrow 0$, the subsequence $u_{\varepsilon_{k}}$ converges strongly to $u_{p}$ in $W^{1, p}\left(B_{1}\right)$, where $u_{p}$ is a least map of the energy $\int_{B_{1}}|\nabla u|^{p} d x$ on $W_{g}^{1, p}\left(B_{1}, S^{n-1}\right)$. 
(2) In Case I with $p>n$ or in Case II, according to Theorem 1 in [Misawa 2001], we can conclude that for some subsequence $u_{\varepsilon_{k}}$, as $k \rightarrow \infty$, the subsequence $u_{\varepsilon_{k}}$ converges weakly to $u_{p}$ in $W^{1, p}(K)$, where $u_{p}$ is a p-harmonic map on $B_{1} \backslash\left\{0, a_{1}, a_{2}, \ldots, a_{N}\right\}$. Its proof is also similar to that of Theorem 1.2 in [Hong 1996, pp632-633].

(3) When $p>2 n-2$, from [Lei and Wu 2000, §6] we can deduce that for some subsequence $\tilde{u}_{k}$ of the regularized minimizer $\tilde{u}_{\varepsilon}$ introduced in [Hong 1996], if $k$ tends to $\infty$, then $\tilde{u}_{k} \rightarrow u_{p}$ in $C^{1, \alpha}(K), \alpha \in(0,1)$, where $u_{p}$ is a p-harmonic map on $B_{1} \backslash\left\{0, a_{1}, a_{2}, \ldots, a_{N}\right\}$.

Now, we shall loosen the constraint $p>2 n-2$. The following theorem will be proved in Section 5.

Theorem 1.3. Assume $\tilde{u}_{\varepsilon}$ is a regularized minimizer and let $K$ be any compact subset of $B_{1} \backslash\left\{0, a_{1}, a_{2}, \ldots, a_{N}\right\}$. When $p \neq n$ and $p>n-t$, for $t$ a constant in $(0, \min \{1 / 2,4 / n\})$, if $\varepsilon \rightarrow 0$, then there is a subsequence $\tilde{u}_{k}$ of $\tilde{u}_{\varepsilon}$ such that

$$
\tilde{u}_{k} \rightarrow u_{p} \text { in } C^{1, \alpha}(K), \quad \alpha \in(0,1),
$$

where $u_{p}$ is a p-harmonic map on $B_{1} \backslash\left\{0, a_{1}, a_{2}, \ldots, a_{N}\right\}$.

Remark 3. Via the uniform estimation in this paper, we prove the convergence of $u_{\varepsilon}$. The compactness only leads to the convergence for some subsequence. If the limit $u_{p}$ is unique, the convergence can be verified for the whole sequence. However, the uniqueness of $u_{p}$ is yet to be established.

When $p=n$, all the results above can still be deduced by analogous arguments in [Ding et al. 1998; Han and Li 1996; Hong 1996; Lei 2004].

\section{Preliminaries}

Proposition 2.1. The minimizer $u_{\varepsilon} \in W$ satisfies

$(2-1)$

$$
\int_{B_{1}}|\nabla u|^{p-2} \nabla u \nabla \phi d x-\frac{1}{\varepsilon^{p}} \int_{B_{1} \backslash B_{\varrho}} u \phi\left(1-|u|^{2}\right) d x+\frac{1}{\varepsilon^{p}} \int_{B_{\varrho}} u \phi|u|^{2} d x=0,
$$

for all $\phi \in W^{1, p}\left(B_{1}, R^{n}\right)$ where $\left.\phi\right|_{\partial B_{1}}=0$. Moreover, $\left|u_{\varepsilon}\right| \leq 1$ a.e. on $\bar{B}_{1}$.

Proof. Using calculus of variations, set $u=u_{\varepsilon}$ in $(2-1)$ and $\phi=u\left(|u|^{2}-1\right)_{+}$, where $\left(|u|^{2}-1\right)_{+}=\min \left(k, \max \left(0,|u|^{2}-1\right)\right)$, for $k$ a positive constant. We then have

$$
\begin{aligned}
\int_{B_{1}}|\nabla u|^{p}\left(|u|^{2}-1\right)_{+} d x+2 \int_{B_{1}}|\nabla u|^{p-2}(u \nabla u)^{2} d x & \\
& +\frac{1}{\varepsilon^{p}} \int_{B_{1} \backslash B_{\varrho}}|u|^{2}\left(|u|^{2}-1\right)_{+}^{2} d x+\frac{1}{\varepsilon^{p}} \int_{B_{\varrho}}|u|^{4}\left(|u|^{2}-1\right)_{+} d x=0,
\end{aligned}
$$


from which it follows that

$$
\frac{1}{\varepsilon^{p}} \int_{B_{1} \backslash B_{\varrho}}|u|^{2}\left(|u|^{2}-1\right)_{+}^{2} d x+\frac{1}{\varepsilon^{p}} \int_{B_{\varrho}}|u|^{4}\left(|u|^{2}-1\right)_{+} d x=0 .
$$

Thus $|u|=0$ or $\left(|u|^{2}-1\right)_{+}=0$ a.e. on $B_{1}$, and hence $|u| \leq 1$ a.e. on $\bar{B}_{1}$.

Proposition 2.2. Assume that $u_{\varepsilon} \in W$ satisfies (2-1). Then for any $\rho>0$, there exists a positive constant $C_{1}$ independent of $\varrho$ and $\varepsilon$, such that for $x \in B(0,1-\rho \varepsilon)$,

$$
\left\|\nabla u_{\varepsilon}(x)\right\|_{L^{\infty}(B(x, \rho \varepsilon))} \leq C_{1} \varepsilon^{-1} .
$$

Proof. Let $y=x \varepsilon^{-1}$ in $(2-1)$ and set $v(y)=u(x)$. Then for any $\phi \in W_{0}^{1, p}\left(B_{\varepsilon}, R^{n}\right)$, we have

$$
\begin{aligned}
\int_{B_{\varepsilon^{-1}}}|\nabla v|^{p-2} \nabla v & \nabla \phi d y \\
& =\int_{B_{\varepsilon^{-1}} \backslash B\left(0, \varrho \varepsilon^{-1}\right)} v\left(1-|v|^{2}\right) \phi d y-\int_{B\left(0, \varrho \varepsilon^{-1}\right)} v \phi|v|^{2} d y .
\end{aligned}
$$

Taking $\phi=v \zeta^{p}$ for some $\zeta \in C_{0}^{\infty}\left(B_{\varepsilon}, R\right)$, we obtain the inequality

$$
\begin{aligned}
& \int_{B_{\varepsilon^{-1}}}|\nabla v|^{p} \zeta^{p} d y \leq p \int_{B_{\varepsilon^{-1}}}|\nabla v|^{p-1} \zeta^{p-1}|\nabla \zeta||v| d y \\
& +\int_{B_{\varepsilon^{-1}} \backslash B\left(0, \varrho \varepsilon^{-1}\right)}|v|^{2}\left(1-|v|^{2}\right) \zeta^{p} d y+\int_{B\left(0, \varrho \varepsilon^{-1}\right)}\left|v^{4}\right| \zeta^{p} d y .
\end{aligned}
$$

Choose $y \in B\left(0, \varepsilon^{-1}-\rho\right)$ such that $B(y, 4 \rho) \subset B_{\varepsilon^{-1}}$. Taking $\zeta=1$ in $B(y, 2 \rho)$ and $\zeta=0$ in $B_{\varepsilon^{-1}} \backslash B(y, 4 \rho)$ satisfying $|\nabla \zeta| \leq C(\rho)$, we have

$$
\int_{B(y, 4 \rho)}|\nabla v|^{p} \zeta^{p} \leq C \int_{B(y, 4 \rho)}|\nabla v|^{p-1} \zeta^{p-1}+C .
$$

Using Hölder's inequality, we can derive that $\int_{B(y, 2 \rho)}|\nabla v|^{p} \leq C$. Combining this with [1983, p. 244, lines 19-23] yields that

$$
\|\nabla v\|_{L^{\infty}(B(y, \rho))}^{p} \leq C \int_{B(y, 2 \rho)}(1+|\nabla v|)^{p} \leq C .
$$

Setting $x=y \varepsilon$ in this inequality completes the proof.

Proposition 2.3. Let $u_{\varepsilon}$ be a minimizer. Then there is a constant $C_{2}>0$ which is independent of $\varrho, \varepsilon \in(0,1)$, such that

$$
\begin{array}{ll}
E_{\varepsilon}\left(u_{\varepsilon}, B_{1}\right) \leq C_{2}\left(1+\varepsilon^{n-p}\right) & \text { in Case I, } \\
E_{\varepsilon}\left(u_{\varepsilon}, B_{1}\right) \leq C_{2}\left(1+\varepsilon^{n-p}+\varrho^{n-1} \varepsilon^{1-p}\right) & \text { in Case II. }
\end{array}
$$


Proof. In Case I, let $y=x \varepsilon^{-1}$. Then

$E_{\varepsilon}\left(u_{\varepsilon}, B_{1}\right)=\frac{1}{2} \int_{B_{\varepsilon^{-1}}}\left|\nabla u_{\varepsilon}\right|^{p} d y+\frac{1}{4} \int_{B_{\varepsilon^{-1}} \backslash B_{\rho \varepsilon^{-1}}}\left(1-\left|u_{\varepsilon}\right|^{2}\right)^{2} d y+\frac{1}{2} \int_{B_{\rho \varepsilon^{-1}}}\left|u_{\varepsilon}\right|^{2} d y$.

Clearly, there exists $u_{1} \in W$ minimizing

$$
F\left(u, B_{1}\right)=\frac{1}{2} \int_{B_{1}}|\nabla u|^{p} d y+\frac{1}{4} \int_{B_{1}}\left(1-|u|^{2}\right)^{2} d y+\frac{1}{2} \int_{B_{1}}|u|^{2} d y .
$$

Define

$$
u_{2}= \begin{cases}u_{1}, & \text { if } 0<|y|<1 \\ \frac{y}{|y|}, & \text { if } 1 \leq|y| \leq \varepsilon^{-1}\end{cases}
$$

Noticing that $u_{\varepsilon}$ is a minimizer of $E_{\varepsilon}\left(u, B_{1}\right)$, we have

$$
E_{\varepsilon}\left(u_{\varepsilon}, B_{1}\right) \leq E_{\varepsilon}\left(u_{2}, B_{1}\right)=F\left(u_{1}, B_{1}\right)+\frac{1}{2} \int_{B_{\varepsilon^{-1}} \backslash B_{1}}\left|\nabla \frac{y}{|y|}\right|^{p} d y \leq C_{2}\left(1+\varepsilon^{n-p}\right) .
$$

In Case II, assume $\varrho<1 / 4$. For any integer $1 \leq j \leq|d|$, take disjoint balls $\left\{B\left(x_{i}, R_{0}\right)\right\}$ for $1 \leq i \leq j$ in $B_{1} \backslash B_{1 / 2}$, where $R_{0}$ is a sufficiently small constant. Set

$$
w(x)= \begin{cases}v(x), & \text { if } x \in\left(B_{1} \backslash B_{1 / 2}\right) \backslash\left(\cup_{i=1}^{j} B\left(x_{i}, R_{0}\right)\right) ; \\ \left(x-x_{i}\right) /\left|x-x_{i}\right|, & \text { if } x \in B\left(x_{i}, R_{0}\right) \backslash B\left(x_{i}, \varepsilon R_{0}\right), 1 \leq i \leq j ; \\ v_{i}(x), & \text { if } x \in B\left(x_{i}, \varepsilon R_{0}\right), 1 \leq i \leq j ; \\ (x /|x|)^{|d|-j}, & \text { if } x \in B_{1 / 2} \backslash B_{\varrho+\varepsilon} ; \\ (|x|-\varrho / \varepsilon)(x /|x|)^{|d|-j}, & \text { if } x \in B_{\varrho+\varepsilon} \backslash B_{\varrho} ; \\ 0, & \text { if } x \in B_{\varrho},\end{cases}
$$

where $(x /|x|)^{m}$, for $m$ a positive integer, is the $S^{n-1}$-valued map given in $n$ dimensional ball coordinates by $(x /|x|)^{m}=\left(\cos m \theta_{1}, \sin m \theta_{1} \cos m \theta_{2}, \ldots\right.$, $\left.\sin m \theta_{1} \cdots \sin m \theta_{n-2} \cos m \theta_{n-1}, \sin m \theta_{1} \cdots \sin m \theta_{n-1}\right)$,

where $v \in W^{1, p}\left(\left(B_{1} \backslash B_{1 / 2}\right) \backslash\left(\bigcup_{i=1}^{j} B\left(x_{i}, R_{0}\right)\right), S^{n-1}\right)$ satisfies

$$
v\left|\partial B_{1}=g, \quad v\right| \partial B_{1 / 2}=(x /|x|)^{|d|-j} \text {, and } \quad v\left|\partial B\left(x_{i}, R_{0}\right)=x /\right| x \mid, \quad 1 \leq i \leq j,
$$

and where $v_{i}$ is a minimizer of $E_{\varepsilon}\left(u, B\left(x_{i}, \varepsilon R_{0}\right)\right)$ in $W^{1, p}\left(B\left(x_{i}, \varepsilon R_{0}\right), R^{n}\right)$ satisfying

$$
\left.v_{i} \mid \partial B\left(x_{i}, \varepsilon R_{0}\right)\right)=\left(x-x_{i}\right) /\left|x-x_{i}\right|, \quad 1 \leq i \leq j .
$$


By calculating, we have

$$
\begin{gathered}
E_{\varepsilon}\left(v,\left(B_{1} \backslash B_{1 / 2}\right) \backslash\left(\bigcup_{i=1}^{j} B\left(x_{i}, R_{0}\right)\right)\right) \leq C ; \\
E_{\varepsilon}\left(w, B\left(x_{i}, R_{0}\right) \backslash B\left(x_{i}, \varepsilon R_{0}\right)\right) \leq C\left(1+\varepsilon^{n-p}\right) ; \\
E_{\varepsilon}\left(v_{i}, B\left(x_{i}, \varepsilon R_{0}\right)\right) \leq C\left(1+\varepsilon^{n-p}\right) .
\end{gathered}
$$

In addition,

$$
\begin{aligned}
E_{\varepsilon}\left(w, B_{1 / 2} \backslash B_{\varrho+\varepsilon}\right) & =\frac{1}{p} \int_{B_{1 / 2} \backslash B_{\varrho+\varepsilon}}\left|\nabla\left(\frac{x}{|x|}\right)^{|d|-j}\right|^{p} d x \\
& =\frac{(n-1)^{p / 2}}{p}(|d|-j)^{p}\left|S^{n-1}\right| \int_{\varrho+\varepsilon}^{1 / 2} r^{n-p-1} d r \leq C\left(1+\varepsilon^{n-p}\right) .
\end{aligned}
$$

Since $0 \leq(r-\varrho) / \varepsilon \leq 1$ on $[\varrho, \varrho+\varepsilon]$, we obtain

$$
\begin{aligned}
E_{\varepsilon}\left(w, B_{\varrho+\varepsilon} \backslash B_{\varrho}\right)= & \frac{1}{p} \int_{B_{\varrho+\varepsilon} \backslash B_{\varrho}}\left(\left|\nabla \frac{|x|-\varrho}{\varepsilon}\right|^{2}+\left(\frac{|x|-\varrho}{\varepsilon}\left|\nabla\left(\frac{x}{|x|}\right)^{|d|-j}\right|^{2}\right)^{p / 2} d x\right. \\
& \quad+\frac{1}{4 \varepsilon^{p}} \int_{B_{\varrho+\varepsilon} \backslash B_{\varrho}}\left(1-\left(\frac{|x|-\varrho}{\varepsilon}\right)^{2}\right)^{2} d x \\
& \leq \frac{C}{\varepsilon^{p}} \int_{\varepsilon}^{\varrho+\varepsilon} r^{n-1} d r \leq C \varepsilon^{-p}\left((\varrho+\varepsilon)^{n}-\varepsilon^{n}\right) \leq C \varrho^{n-1} \varepsilon^{1-p} .
\end{aligned}
$$

Combining these estimates and noting that $u_{\varepsilon}$ is a minimizer, we have

$$
E_{\varepsilon}\left(u_{\varepsilon}, B_{1}\right) \leq E_{\varepsilon}\left(w, B_{1}\right) \leq C_{2}\left(\varepsilon^{n-p}+\varrho^{n-1} \varepsilon^{1-p}\right) .
$$

Proposition 2.4. In Case II, for any given $\gamma>1$, there exists $C_{3}>0$ which is independent of $\varrho, \varepsilon \in(0,1)$, such that

$$
E_{\varepsilon}\left(u_{\varepsilon}, B_{1} \backslash B_{\gamma \varrho}\right) \leq C_{3}\left(1+\varepsilon^{n-p}\right) .
$$

Proof. We prove the proposition by means of induction. Set

$$
w_{1}= \begin{cases}w, & \text { if } x \in B_{1} \backslash B_{1 / 2} ; \\ (x /|x|)^{|d|-j}, & \text { if } x \in B_{1 / 2} \backslash B_{\varepsilon} ; \\ (|x| / \varepsilon)(x /|x|)^{|d|-j}, & \text { if } x \in B_{\varepsilon} .\end{cases}
$$

For any $\gamma>1$, there exists $\delta>0$ such that $\gamma-\delta>1$. According to Proposition 2.3, we have

$$
E_{\varepsilon}\left(u_{\varepsilon}, B_{1} \backslash B_{\left(\gamma-\delta+(k-1) n^{-1} \delta\right) \varrho}\right) \leq C\left(1+\varepsilon^{n-p}+\varrho^{n-k} \varepsilon^{k-p}\right)
$$

with $k=1$. Suppose (2-3) holds for $k=m$ with $m=2,3, \ldots, n-1$. Then we shall verify it for $k=m+1$. 
By the mean value theorem, there is $\sigma_{m} \in\left(\gamma-\delta+(m-1) n^{-1} \delta, \gamma-\delta+m n^{-1} \delta\right)$ such that

$$
\begin{aligned}
\int_{B_{\gamma \varrho} \backslash B_{(\gamma-\delta) \varrho}}\left(1-\left|u_{\varepsilon}\right|^{2}\right)^{2} d x & =\int_{(\gamma-\delta) \varrho}^{\gamma \varrho}\left(\int_{S^{n-1}}\left(1-\left|u_{\varepsilon}(r, \zeta)\right|^{2}\right)^{2} d \zeta\right) r^{n-1} d r \\
& =\int_{S^{n-1}}\left(1-\left|u_{\varepsilon}\left(\sigma_{m} \varrho, \zeta\right)\right|^{2}\right)^{2} d \zeta \cdot \int_{(\gamma-\delta) \varrho}^{\gamma \varrho} r^{n-1} d r \\
& =C^{-1} \varrho^{n} \int_{S^{n-1}}\left(1-\left|u_{\varepsilon}\left(\sigma_{m} \varrho, \zeta\right)\right|^{2}\right)^{2} d \zeta .
\end{aligned}
$$

This means that

$$
\begin{aligned}
\frac{1}{\varepsilon^{p}} \int_{S^{n-1}}\left(1-\left|u_{\varepsilon}\left(\sigma_{m} \varrho, \zeta\right)\right|^{2}\right)^{2} d \zeta & =C \varrho^{-n} \varepsilon^{-p} \int_{B_{\gamma \varrho} \backslash B_{(\gamma-\delta) \varrho}}\left(1-\left|u_{\varepsilon}\right|^{2}\right)^{2} d x \\
& \leq C \varrho^{-n}\left(1+\varepsilon^{n-p}+\varrho^{n-m} \varepsilon^{m-p}\right),
\end{aligned}
$$

by applying (2-3) with $k=m$. Define

$$
\bar{u}(r)=\left(\frac{1}{\left|S^{n-1}\right|} \int_{S^{n-1}}|u(r, \zeta)|^{2} d \zeta\right)^{1 / 2} \quad \text { for } r \in[0,1] .
$$

Using Hölder's inequality, we easily get

$$
\begin{gathered}
\left(1-|\bar{u}(r)|^{2}\right)^{2} \leq \frac{1}{\left|S^{n-1}\right|} \int_{S^{n-1}}\left(1-|u(r, \zeta)|^{2}\right)^{2} d \zeta \quad \text { for } r \in[0,1], \\
\left|\frac{d \bar{u}(r)}{d r}\right|^{2} \leq \frac{1}{\left|S^{n-1}\right|} \int_{S^{n-1}}\left|\frac{\partial u(r, \zeta)}{\partial r}\right|^{2} d \zeta \quad \text { a.e. } r \in[0,1] .
\end{gathered}
$$

Let

$$
W_{m}= \begin{cases}w_{1}, & \text { if } x \in B_{1} \backslash B_{\sigma_{m} \varrho+\varepsilon} \\ \left(|x|-\sigma_{m} \varrho / \varepsilon\right)\left(1-\bar{u}\left(\sigma_{m} \varrho\right)\right)+\bar{u}\left(\sigma_{m} \varrho\right) w_{1}, & \text { if } x \in B_{\sigma_{m} \varrho+\varepsilon} \backslash B_{\sigma_{m} \varrho} \\ \bar{u} w_{1}, & \text { if } x \in B_{\sigma_{m} \varrho} .\end{cases}
$$

Obviously,

$$
\int_{B_{1}}\left|\nabla w_{1}\right|^{p}+E_{\varepsilon}\left(w_{1}, B_{1} \backslash B_{\sigma_{m} \varrho+\varepsilon}\right) \leq C\left(1+\varepsilon^{n-p}\right) .
$$

From (2-4), (2-5), and $\varepsilon \varrho^{-1} \leq 1$, it follows that

$$
\begin{aligned}
J_{m} & :=\frac{1}{\varepsilon^{p}} \int_{\sigma_{m} \varrho}^{\sigma_{m} \varrho+\varepsilon}\left(1-\left|\bar{u}\left(\sigma_{m} \varrho\right)\right|^{2}\right)^{2} r^{n-1} d r \\
& \leq C \varrho^{-n}\left(1+\varepsilon^{n-p}+\varrho^{n-m} \varepsilon^{m-p}\right) \int_{\sigma_{m} \varrho}^{\sigma_{m} \varrho+\varepsilon} r^{n-1} d r \\
& \leq C \varrho^{-n}\left(1+\varepsilon^{n-p}+\varrho^{n-m} \varepsilon^{m-p}\right) \varepsilon \varrho^{n-1} \leq C\left(1+\varepsilon^{n-p}+\varrho^{n-m-1} \varepsilon^{m+1-p}\right) .
\end{aligned}
$$


So since $0 \leq\left(r-\sigma_{m} \varrho\right) / \varepsilon \leq 1$ for $r \in\left[\sigma_{m} \varrho, \sigma_{m} \varrho+\varepsilon\right]$, by $(2-7)$ we have

$(2-8) \quad E_{\varepsilon}\left(W_{m}, B_{\sigma_{m} \varrho+\varepsilon} \backslash B_{\sigma_{m} \varrho}\right)$

$$
\begin{aligned}
\leq & C \int_{B_{\sigma_{m} \varrho+\varepsilon} \backslash B_{\sigma_{m} \varrho}}\left(\left|\nabla w_{1}\right|^{p}\left(\left|\bar{u}\left(\sigma_{m} \varrho\right)\right|+\frac{|x|-\sigma_{m} \varrho}{\varepsilon}\left(1-\left|\bar{u}\left(\sigma_{m} \varrho\right)\right|\right)\right)^{p}\right. \\
& \left.+\left(\frac{1-\left|\bar{u}\left(\sigma_{m} \varrho\right)\right|}{\varepsilon}\right)^{p}|\nabla| x||^{p}\right) d x \\
& +\frac{1}{4 \varepsilon^{p}} \int_{B_{\sigma_{m} \varrho+\varepsilon} \backslash B_{\sigma_{m}}}\left(1-\left(\left|\bar{u}\left(\sigma_{m} \varrho\right)\right|+\frac{|x|-\sigma_{m} \varrho}{\varepsilon}\left(1-\left|\bar{u}\left(\sigma_{m} \varrho\right)\right|\right)\right)^{2}\right)^{2} \\
\leq & C \int_{B_{\sigma_{m} \varrho+\varepsilon} \backslash B_{\sigma_{m} \varrho}}\left|\nabla w_{1}\right|^{p}+C J_{m} \leq C\left(1+\varepsilon^{n-p}+\varrho^{n-m-1} \varepsilon^{m+1-p}\right) .
\end{aligned}
$$

Next, by the definition of $w_{1}$ and $W_{m}$ and from (2-5) and (2-6), it follows that

$$
E_{\varepsilon}\left(W_{m}, B_{\sigma_{m} \varrho} \backslash B_{\varepsilon}\right) \leq E_{\varepsilon}\left(u_{\varepsilon}, B_{\sigma_{m} \varrho} \backslash B_{\varepsilon}\right) .
$$

Finally, on $B_{\varepsilon}$,

(2-10) $\left(|\nabla \bar{u}|^{2}+|\bar{u}|^{2}\left|\nabla w_{1}\right|^{2}\right)^{p / 2}=\left(|\nabla \bar{u}|^{2}+|\bar{u}|^{2} \frac{|x|^{2}}{\varepsilon^{2}}\left|\nabla\left(\frac{x}{|x|}\right)^{|d|-j}\right|^{2}+\frac{1}{\varepsilon^{2}}|\bar{u}|^{2}\right)^{p / 2}$.

Using the mean value theorem, we see that on $B_{\varepsilon}$,

$$
\begin{aligned}
& \left(|\nabla \bar{u}|^{2}+|\bar{u}|^{2} \frac{|x|^{2}}{\varepsilon^{2}}\left|\nabla\left(\frac{x}{|x|}\right)^{|d|-j}\right|^{2}+\frac{1}{\varepsilon^{2}}|\bar{u}|^{2}\right)^{p / 2}-\left(|\nabla \bar{u}|^{2}\right)^{p / 2} \\
& =\frac{p|\bar{u}|^{2}}{2}\left(\frac{1}{\varepsilon^{2}}+\frac{(|d|-j)^{2}}{\varepsilon^{2}}\right) \int_{0}^{1}\left(s\left(|\nabla \bar{u}|^{2}+|\bar{u}|^{2} \frac{|x|^{2}}{\varepsilon^{2}}\left|\nabla\left(\frac{x}{|x|}\right)^{|d|-j}\right|^{2}+\frac{1}{\varepsilon^{2}}|\bar{u}|^{2}\right)\right. \\
& \left.+(1-s)\left(|\nabla \bar{u}|^{2}\right)\right)^{(p-2) / 2} d s \\
& =\frac{p|\bar{u}|^{2}}{2} \frac{1+(|d|-j)^{2}}{\varepsilon^{2}} I .
\end{aligned}
$$

From Proposition 2.2 and (2-6), it follows that $I \leq C \varepsilon^{2-p}$. Substituting this into the preceding equality and combining with $(2-10)$, we obtain

$$
\begin{aligned}
E_{\varepsilon}\left(\bar{u} w_{1}, B_{\varepsilon}\right)=\frac{1}{p} \int_{B_{\varepsilon}}\left(|\nabla \bar{u}|^{2}+|\bar{u}|^{2}\left|\nabla w_{1}\right|^{2}\right)^{p / 2} d x+\frac{1}{2 \varepsilon^{p}} \int_{B_{\varepsilon}} w_{1}^{2}|\bar{u}|^{2} d x \\
\leq E_{\varepsilon}\left(\bar{u}, B_{\varepsilon}\right)+C \varepsilon^{2-p} \int_{0}^{\varepsilon}|\bar{u}|^{2} \frac{1}{\varepsilon^{2}} r^{n-1} d r .
\end{aligned}
$$

By the definition of $\bar{u}$ and (2-6), we have at last that

$$
E_{\varepsilon}\left(\bar{u} w_{1}, B_{\varepsilon}\right) \leq E_{\varepsilon}\left(u_{\varepsilon}, B_{\varepsilon}\right)+C \varepsilon^{n-p} .
$$


Since $u_{\varepsilon}$ is a minimizer, it follows from (2-7)-(2-9) and (2-11) that

$$
E_{\varepsilon}\left(u_{\varepsilon}, B_{1}\right) \leq E_{\varepsilon}\left(W_{m}, B_{1}\right) \leq C\left(1+\varepsilon^{n-p}+\varrho^{n-m-1} \varepsilon^{m+1-p}\right)+E_{\varepsilon}\left(u_{\varepsilon}, B_{\sigma_{m}} \varrho\right) .
$$

Observing that $E_{\varepsilon}\left(u_{\varepsilon}, B_{1} \backslash B_{\left(\gamma-\delta+m n^{-1} \delta\right) \varrho}\right) \leq E_{\varepsilon}\left(u_{\varepsilon}, B_{1} \backslash B_{\sigma_{m} \varrho}\right)$, we see that (2-3) holds with $k=m+1$. Proposition 2.4 follows by taking $k=n$ in (2-3).

\section{Location of zeros}

At first, we will show that there is no zero of $u_{\varepsilon}$ near the boundary $\partial B_{1}$.

Theorem 3.1. There is a constant $\rho>0$, such that for $x \in B_{1} \backslash B_{1-2 \rho \varepsilon}$,

$$
\left|u_{\varepsilon}(x)\right| \geq 1 / 2 \text {. }
$$

Proof. Scaling $y=x \varepsilon^{-1}$ in (2-1) yields (2-2). According to the results of the $C^{\alpha}$ estimation of $v$ (see, for example, Theorem 1 and lines 19-21 on p. 104 of [Chen and DiBenedetto 1989]), there exist $C>0$ and $\alpha \in(0,1)$, such that for any $\rho \in(0,1)$ and $y_{0} \in \partial B_{\varepsilon^{-1}}$, we have $\left|v(y)-v\left(y_{0}\right)\right| \leq C\left|y-y_{0}\right|^{\alpha}$ for all $y \in B_{\varepsilon^{-1}} \cap B\left(y_{0}, 4 \rho\right)$. Taking $\rho=1 /(8 C)$, we obtain $|v(y)| \geq\left|v\left(y_{0}\right)\right|-C\left|y-y_{0}\right| \geq 1 / 2$. Letting $x=y \varepsilon$, easily implies the theorem.

Proposition 3.2. Let $u_{\varepsilon}$ be a minimizer of $E_{\varepsilon}\left(u, B_{1}\right)$. There exists a constant $C>0$ which is independent of $\varrho, \varepsilon \in\left(0, \varepsilon_{0}\right)$ with $\varepsilon_{0}$ sufficiently small, such that

$$
\begin{aligned}
\frac{1}{\varepsilon^{n}} \int_{B_{1} \backslash B_{\varrho}}\left(1-\left|u_{\varepsilon}\right|^{2}\right)^{2}+\frac{1}{\varepsilon^{n}} \int_{B_{\varrho}}\left|u_{\varepsilon}\right|^{2} \leq C \quad \text { in Case I, } \\
\frac{1}{\varepsilon \varrho^{n-1}} \int_{B_{1} \backslash B_{\varrho}}\left(1-\left|u_{\varepsilon}\right|^{2}\right)^{2}+\frac{1}{\varepsilon \varrho^{n-1}} \int_{B_{\varrho}}\left|u_{\varepsilon}\right|^{2} \leq C \quad \text { in Case II. }
\end{aligned}
$$

Furthermore, in Case II with $p>n$, for any $\gamma>1$, there is $C>0$ independent of $\varrho, \varepsilon \in\left(0, \varepsilon_{0}\right)$ such that

$$
\frac{1}{\varepsilon^{n}} \int_{B_{1} \backslash B_{\gamma \varrho}}\left(1-\left|u_{\varepsilon}\right|^{2}\right)^{2} \leq C .
$$

Proof. When $p>n,(3-1)-(3-3)$ are corollaries of Proposition 2.3 and Proposition 2.4 by multiplying by $\varepsilon^{p-n}$ or $\varepsilon^{p-1} \varrho^{1-n}$. When $1<p<n$, the idea of the proof comes from [Struwe 1993]. Set $v[\varepsilon]=\inf \left\{E_{\varepsilon}\left(u, B_{1}\right) ; u \in W\right\}$. For fixed $u \in W$, the map $\varepsilon \rightarrow E_{\varepsilon}\left(u, B_{1}\right)$ is nonincreasing, and

$$
-\frac{\partial}{\partial \varepsilon} E_{\varepsilon}\left(u, B_{1}\right)=\frac{p}{4 \varepsilon^{p+1}} \int_{B_{1} \backslash B_{\varrho}}\left(1-\left|u_{\varepsilon}\right|^{2}\right)^{2}+\frac{p}{2 \varepsilon^{p+1}} \int_{B_{\varrho}}\left|u_{\varepsilon}\right|^{2} .
$$


Noting $v[\varepsilon+\delta] \leq E_{\varepsilon+\delta}\left(u_{\varepsilon}, B_{1}\right) \leq E_{\varepsilon}\left(u_{\varepsilon}, B_{1}\right)=v[\varepsilon]$, we have

$$
\begin{aligned}
& \frac{1}{4 \varepsilon^{p+1}} \int_{B_{1} \backslash B_{\varrho}}\left(1-\left|u_{\varepsilon}\right|^{2}\right)^{2}+\frac{1}{2 \varepsilon^{p+1}} \int_{B_{\varrho}}\left|u_{\varepsilon}\right|^{2} \\
& \quad=\lim _{\delta \rightarrow 0} \frac{E_{\varepsilon}\left(u_{\varepsilon}, B_{1}\right)-E_{\varepsilon+\delta}\left(u_{\varepsilon}, B_{1}\right)}{\delta} \leq \varlimsup_{\delta \rightarrow 0} \frac{\nu[\varepsilon]-v[\varepsilon+\delta]}{\delta}=-v^{\prime}[\varepsilon] .
\end{aligned}
$$

We claim that there exists a constant $M>0$ independent of $\varepsilon$ and $\varrho$ such that when both $\varrho$ and $\varepsilon$ tend to zero,

$$
\begin{array}{ll}
-\varepsilon^{p+1-n} \nu^{\prime}[\varepsilon] \leq M & \text { in Case I; } \\
-\varepsilon^{p} \varrho^{1-n} \nu^{\prime}[\varepsilon] \leq M & \text { in Case II. }
\end{array}
$$

Otherwise, we can find $\varepsilon_{1}>0$, such that if $\varepsilon, \varrho \in\left(0, \varepsilon_{1}\right)$, then $-v^{\prime}[\varepsilon]>M \varepsilon^{n-p-1}$ in Case I, and $-v^{\prime}[\varepsilon]>M \varepsilon^{-p} \varrho^{n-1}$ in Case II. Now, let $M=2(n-p)\left(C_{2}+1\right) \varepsilon_{1}^{p-n}$ in Case I and $M=2\left(C_{2}+1\right)(p-1)$ in Case II. Here, $C_{2}$ is the constant in Proposition 2.3. Integrating from $\varepsilon$ to $\varepsilon_{1}$, we obtain

$$
\begin{array}{ll}
\nu[\varepsilon] \geq \nu\left[\varepsilon_{1}\right]-\int_{\varepsilon}^{\varepsilon_{1}} \nu^{\prime}[\varepsilon] d \varepsilon>v\left[\varepsilon_{1}\right]+2 C_{2}+1 & \text { in Case I; } \\
\nu[\varepsilon] \geq \nu\left[\varepsilon_{1}\right]-\int_{\varepsilon}^{\varepsilon_{1}} \nu^{\prime}[\varepsilon] d \varepsilon>v\left[\varepsilon_{1}\right]+\left(2 C_{2}+1\right) \varepsilon^{1-p} \varrho^{n-1} & \text { in Case II. }
\end{array}
$$

These contradict Proposition 2.3. Substituting (3-5) into (3-4), we can find a sufficiently small $\varepsilon_{0}>0$, such that (3-1) and (3-2) hold with $C=M+1$.

Hereafter, we assume $\varepsilon, \varrho \in\left(0, \varepsilon_{0}\right)$. For any $\gamma>1$, set

$$
\begin{array}{ll}
A=B_{1-\rho \varepsilon} \backslash B_{\gamma \varrho} & \text { in Case II, } \\
A=B_{1-\rho \varepsilon} \backslash B_{\varrho} & \text { in Case I. }
\end{array}
$$

Proposition 3.3. Let $u_{\varepsilon}$ be a minimizer of $E_{\varepsilon}\left(u, B_{1}\right)$. Then for any $\eta \in(0,1 / 2)$, there exist positive constants $\lambda, \mu$ which are independent of $\varrho, \varepsilon$, such that

(1) In Case I or in Case II with $p>n$, if

$$
\frac{1}{\varepsilon^{n}} \int_{A \cap B(\cdot, 2 l \varepsilon)}\left(1-\left|u_{\varepsilon}\right|^{2}\right)^{2} \leq \mu,
$$

for $B(\cdot, 2 l \varepsilon)$ a ball of radius $2 l \varepsilon$ with $l \geq \lambda$, then $\left|u_{\varepsilon}(x)\right| \geq 1-\eta$ for all $x \in$ $A \cap B(\cdot, l \varepsilon)$.

(2) In Case I, if

$$
\frac{1}{\varepsilon^{n}} \int_{B_{\varrho} \cap B(\cdot, 2 l \varepsilon)}\left|u_{\varepsilon}\right|^{2} \leq \mu,
$$

then $\left|u_{\varepsilon}(x)\right| \leq \eta$ for all $x \in B_{\varrho} \cap B(\cdot, l \varepsilon)$. 
Proof. Observe that there exists a constant $C_{3}>0$ which is independent of $\varrho$ and $\varepsilon$, such that for $0<r \leq 1$, if $x$ is in $B_{1}$, then $\left|B_{1} \cap B(x, r)\right| \geq|A \cap B(x, r)| \geq C_{3} r^{n}$. Let $\lambda=\eta /\left(2 C_{1}\right)$ and $\mu=C_{3} \eta^{2} \lambda^{n} / 4$.

Suppose that there is a point $x_{0} \in A \cap B(\cdot, l \varepsilon)$ such that $\left|u_{\varepsilon}\left(x_{0}\right)\right|<1-\eta$. According to Proposition 2.2, we have

$$
\left|u_{\varepsilon}(x)-u_{\varepsilon}\left(x_{0}\right)\right| \leq C_{1} \varepsilon^{-1}\left|x-x_{0}\right|=C_{1} \lambda=\eta / 2 \text { for all } x \in B\left(x_{0}, \lambda \varepsilon\right),
$$

and hence $\left(1-\left|u_{\varepsilon}(x)\right|^{2}\right)^{2}>\eta^{2} / 4$ for all $x \in B\left(x_{0}, \lambda \varepsilon\right)$. Thus

$$
\int_{B\left(x_{0}, \lambda \varepsilon\right) \cap A}\left(1-\left|u_{\varepsilon}\right|^{2}\right)^{2}>\left(\eta^{2} / 4\right)\left|A \cap B\left(x_{0}, \lambda \varepsilon\right)\right| \geq C_{3} \eta(\lambda \varepsilon)^{n} / 4=\mu \varepsilon^{n} .
$$

Since $x_{0} \in B(\cdot, l \varepsilon) \cap A$ and $\left(B\left(x_{0}, \lambda \varepsilon\right) \cap A\right) \subset(B(\cdot, 2 l \varepsilon) \cap A)$, it follows that

$$
\int_{B(\cdot, 2 l \varepsilon) \cap A}\left(1-\left|u_{\varepsilon}\right|^{2}\right)^{2}>\mu \varepsilon^{n},
$$

which contradicts (3-6). This proves (1), and the proof of (2) is analogous.

In Case II with $p \in(1, n)$, Proposition 2.2 is not sufficient to deduce Proposition 3.3. The reason is that in Case II, the estimation (3-2) is not accurate as (3-1), which forces us to investigate (3-8) on the larger ball $B\left(x_{0}, \lambda \varepsilon^{1 / n} \varrho^{1-1 / n}\right)$. Proposition 2.2 is invalid since it only holds on the smaller ball $B\left(x_{0}, \lambda \varepsilon\right)$. To obtain Proposition 3.3, we instead use Proposition 3.4, though it only holds for $p$ sufficiently close to the dimension $n$.

Proposition 3.4. Assume $u_{\varepsilon}$ is a minimizer of $E_{\varepsilon}\left(u, B_{1}\right)$. Then in Case II with $p \in(n-t, n)$ where $t \in(0, \min \{1 / 2,4 / n\})$, there exists a constant $C>0$ such that for any $x, x_{0} \in \bar{A}$,

$$
\left|u_{\varepsilon}(x)-u_{\varepsilon}\left(x_{0}\right)\right| \leq C\left|x-x_{0}\right|^{\alpha} \quad \text { for some } \alpha \in(0,1-n /(p+t)) .
$$

Proof. By the Reverse Hölder inequality (Proposition 3.5) and Proposition 2.4, we have $\left\|\nabla u_{\varepsilon}\right\|_{L^{p+t}(A)} \leq C\left\|\nabla u_{\varepsilon}\right\|_{L^{p}(A)} \leq C$ for some $t \in(0, \min \{1 / 2,4 / n\})$.

Since $\left|u_{\varepsilon}\right| \leq 1$ a.e. on $B$, we obtain $\left\|u_{\varepsilon}\right\|_{W^{1, p+t}(A)} \leq C$. When $p \in(n-t, n)$, by the embedding theorem we see that $\left|u_{\varepsilon}(x)-u_{\varepsilon}\left(x_{0}\right)\right| \leq C\left|x-x_{0}\right|^{\alpha}$ for any $x, x_{0} \in \bar{A}$, for some $\alpha \in(0,1-n /(p+t))$.

Proposition 3.5 (Reverse Hölder inequality). Assume $p>1$ and $u_{\varepsilon}$ is a minimizer of $E_{\varepsilon}\left(u, B_{1}\right)$. Then there exist constants $t \in(0, \min \{1 / 2,4 / n\}), R_{0} \in(0,1 / 2)$ and $C>0$ which are independent of $\varepsilon$ and $\varrho$ such that for any $B(\cdot, R) \subset B_{1}$ with $2 R<R_{0}$,

$\left(\int_{B(\cdot, R)}\left|\nabla u_{\varepsilon}\right|^{q} d x\right)^{1 / q} \leq C\left(\int_{B(\cdot, 2 R)}\left(\left|\nabla u_{\varepsilon}\right|^{2}+1\right)^{p / 2} d x\right)^{1 / p} \quad$ for $q \in[p, p+2 t)$.

The proof is completely analogous to that of Proposition 2.1 in [Lei 2004]. 
Proposition 3.6. Assume $u_{\varepsilon}$ is a minimizer. Then in Case II with $p \in(n-t, n)$ where $t$ is the constant in Proposition 3.5, for any $\eta \in(0,1 / 2)$, there exist positive constants $\lambda, \mu$ which are independent of $\varrho, \varepsilon$, such that if

$$
\frac{1}{\varepsilon \varrho^{n-1}} \int_{A \cap B\left(\cdot, 2 l \varepsilon^{1 / n} \varrho^{(n-1) / n}\right)}\left(1-\left|u_{\varepsilon}\right|^{2}\right)^{2} \leq \mu,
$$

where $B\left(\cdot, 2 l \varepsilon^{1 / n} \varrho^{(n-1) / n}\right)$ is some ball of radius $2 l \varepsilon^{1 / n} \varrho^{(n-1) / n}$ with $l \geq \lambda$, then

$$
\left|u_{\varepsilon}(x)\right| \geq 1-\eta \quad \text { for all } x \in A \cap B\left(\cdot, l \varepsilon^{1 / n} \varrho^{(n-1) / n}\right) .
$$

The proof is like that of Proposition 3.3; the only difference is that we apply Proposition 3.4. instead of Proposition 2.2.

To find the zeros of $u_{\varepsilon}$ in Case I or in Case II with $p>n$, we may take (2-1) as a ruler to distinguish the ball of radius $\lambda \varepsilon$ which contains the zeros. Given $\gamma>1$, let $\lambda, \mu$ be the same constants as in Proposition 3.3. If

$$
\frac{1}{\varepsilon^{n}} \int_{B\left(x^{\varepsilon}, 2 \lambda \varepsilon\right) \cap A}\left(1-\left|u_{\varepsilon}\right|^{2}\right)^{2} \leq \mu,
$$

then $B\left(x^{\varepsilon}, \lambda \varepsilon\right)$ is called a good ball. Otherwise it is called a bad ball. Now suppose that $\left\{B\left(x_{i}^{\varepsilon}, \lambda \varepsilon\right), i \in I\right\}$ is a family of balls satisfying the following conditions:

(i) $x_{i}^{\varepsilon} \in A$ for $i \in I$.

(ii) $A \subset \bigcup_{i \in I} B\left(x_{i}^{\varepsilon}, \lambda \varepsilon\right)$.

(iii) $B\left(x_{i}^{\varepsilon}, \lambda \varepsilon / 4\right) \cap B\left(x_{j}^{\varepsilon}, \lambda \varepsilon / 4\right)=\varnothing$ for $i \neq j$.

Set $J_{\varepsilon}=\left\{i \in I ; B\left(x_{i}^{\varepsilon}, \lambda \varepsilon\right)\right.$ is a bad ball $\}$.

Proposition 3.7. There exists an integer $N$ independent of $\varepsilon$ exceeding the number Card $J_{\varepsilon}$ of bad balls.

Proof. Since (iii) implies that every point in $A$ can be covered by a finite number $m$ of balls where $m$ is independent of $\varepsilon$ and $\varrho$, from (3-1) or (3-3) and the definition of bad ball, we have

$$
\begin{aligned}
\mu \varepsilon^{n} \operatorname{Card} J_{\varepsilon} \leq & \sum_{i \in J_{\varepsilon}} \int_{B\left(x_{i}^{\varepsilon}, 2 \lambda \varepsilon\right) \cap A}\left(1-\left|u_{\varepsilon}\right|^{2}\right)^{2} \\
& \leq m \int_{\bigcup_{i \in J_{\varepsilon}} B\left(x_{i}^{\varepsilon}, 2 \lambda \varepsilon\right) \cap A}\left(1-\left|u_{\varepsilon}\right|^{2}\right)^{2} \leq m \int_{B_{1} \backslash B_{Q}}\left(1-\left|u_{\varepsilon}\right|^{2}\right)^{2} \leq m C \varepsilon^{n} .
\end{aligned}
$$

Hence Card $J_{\varepsilon} \leq m C / \mu \leq N$.

Proof of Theorem 1.1. Based on Proposition 3.7, by applying Theorem IV.1 of [Bethuel et al. 1994], we may modify the family of bad balls so that the new 
family, denoted $\left\{B\left(x_{i}^{\varepsilon}, h \varepsilon\right) ; i \in J\right\}$, satisfies

$$
\bigcup_{i \in J_{\varepsilon}} B\left(x_{i}^{\varepsilon}, \lambda \varepsilon\right) \subset \bigcup_{i \in J} B\left(x_{i}^{\varepsilon}, h \varepsilon\right), \quad \operatorname{Card} J \leq \operatorname{Card} J_{\varepsilon},
$$

and

$$
\left|x_{i}^{\varepsilon}-x_{j}^{\varepsilon}\right|>8 h \varepsilon, \quad i, j \in J, \quad i \neq j,
$$

where $h$ is a constant satisfying $\lambda \leq h=h(\eta) \leq \lambda 9^{N}=9^{N} \eta /\left(2 C_{1}\right)$. Choose $\eta>0$ sufficiently small so that $h<1$. Condition (3-9) implies that no two balls in the new family intersect. Thus the points $x$ where $\left|u_{\varepsilon}(x)\right| \leq 1-\eta$ are contained in these finite, disjoint bad balls $\left\{B\left(x_{i}, h \varepsilon\right)\right\}_{i=1}^{N}$ and $B_{h \varepsilon} \cup B_{\gamma \varrho}$. Combining this with Theorem 3.1, we obtain (1-2).

Similarly, (1-3) is obtained by applying (3-1) and Proposition 3.3(2); (1-4) is obtained by applying (3-3) and Proposition 3.3(1); lastly, (1-5) is obtained by applying (3-2) and Proposition 3.6.

For each $i=1,2, \ldots$, Card $J$, there exists a sequence $\varepsilon_{k} \rightarrow 0$ such that the centers $x_{i}^{\varepsilon_{k}}$ approach either 0 or some $a_{i} \in \bar{B}_{1}$. There may be more than one such subsequence $x_{i}^{\varepsilon_{k}}$ converging to the same point. We denote by $0, a_{1}, a_{2}, \ldots, a_{N}$ the distinct points in $\left\{0, a_{i}\right\}_{i=1}^{\text {Card } J}$.

From the discussion above, we also see that for any $\sigma>0$,

$$
\left|u_{\varepsilon}(x)\right| \geq 1 / 2 \text { for all } x \in \bar{B}_{1} \backslash\left(\bigcup_{j=1}^{\operatorname{Card} J} B\left(a_{j}, \sigma\right) \cup B_{\sigma}\right) .
$$

\section{Uniform estimation}

Let $u_{\varepsilon}$ be a minimizer of $E_{\varepsilon}\left(u, B_{1}\right)$. When $p \in(1, n)$, Propositions 2.3 and 2.4 imply (1-6) and (1-7), respectively. In this section we shall prove (1-7) when $p>n$.

Theorem 4.1. Let $R>0$ be small enough that $B(x, 2 R) \Subset B_{1} \backslash\left\{0, a_{1}, a_{2}, \ldots, a_{N}\right\}$. Then there are constants $C>0$ and $R_{j}=2 R-j R /([p]+1)$ such that

$$
E_{\varepsilon}\left(u_{\varepsilon}, B_{j}\right) \leq C \varepsilon^{j-p}
$$

for $j=n, n+1, \ldots,[p]$, where $\varepsilon \in\left(0, \varepsilon_{0}\right)$ and $B_{j}=B\left(x, R_{j}\right)$.

For $j=n$, the inequality (4-1) is a corollary of Proposition 2.4. Suppose that (4-1) holds for all $j \leq m$. Then, in particular,

$$
E_{\varepsilon}\left(u_{\varepsilon}, B_{m}\right) \leq C \varepsilon^{m-p} .
$$

Suppose $m<[p]$. We want to prove (4-1) for $j=m+1$. 
According to Proposition 2.1 and (3-10), we have $1 / 2 \leq\left|u_{\varepsilon}(x)\right| \leq 1$ for all $x \in B(x, 2 R)$. As in the derivation of (2-4), by (4-2) and the mean value theorem, there is $r \in\left[R_{m+1 / 2}, R_{m}\right]$ such that

$$
\int_{\partial B(x, r)}\left|\nabla u_{\varepsilon}\right|^{p} d \xi+\frac{1}{\varepsilon^{p}} \int_{\partial B(x, r)}\left(1-\left|u_{\varepsilon}\right|^{2}\right)^{2} d \xi \leq C \varepsilon^{m-p} .
$$

Here $\xi$ is the integration variable on $\partial B(x, r)$.

Proposition 4.2. Denote $B(x, r)$ by $B$. If $\rho_{m}$ is a minimizer of the functional

$$
E(\rho, B)=\frac{1}{p} \int_{B}\left(|\nabla \rho|^{2}+1\right)^{p / 2}+\frac{1}{2 \varepsilon^{p}} \int_{B}(1-\rho)^{2}
$$

on $W_{\left|u_{\varepsilon}\right|}^{1, p}\left(B, R^{+} \cup\{0\}\right)$, then $E\left(\rho_{m}, B\right) \leq C \varepsilon^{m-p+1}$.

Proof. Obviously, the minimizer $\rho_{m}$ exists and satisfies

$$
-\operatorname{div}\left(v^{(p-2) / 2} \nabla \rho\right)=1 / \varepsilon^{p}(1-\rho) \quad \text { on } B,
$$

and

$$
\left.\rho\right|_{\partial B}=\left|u_{\varepsilon}\right|,
$$

where $v=|\nabla \rho|^{2}+1$. Since $1 / 2 \leq\left|u_{\varepsilon}\right| \leq 1$, from the maximum principle it follows that

$$
1 / 2 \leq \rho_{m} \leq 1 \quad \text { on } \bar{B} \text {. }
$$

Applying (4-2) we see easily that

$$
E\left(\rho_{m}, B\right) \leq E\left(\left|u_{\varepsilon}\right|, B\right) \leq C E_{\varepsilon}\left(u_{\varepsilon}, B\right) \leq C \varepsilon^{m-p} .
$$

Multiplying (4-4) by $(v \cdot \nabla \rho)$, where $\rho$ denotes $\rho_{m}$, and integrating over $B$, we have

$$
\begin{aligned}
-\int_{\partial B} v^{(p-2) / 2}(v \cdot \nabla \rho)^{2} d \xi+\int_{B} v^{(p-2) / 2} \nabla \rho \cdot \nabla & (v \cdot \nabla \rho) \\
& =\frac{1}{\varepsilon^{p}} \int_{B}(1-\rho)(v \cdot \nabla \rho),
\end{aligned}
$$

where $v$ denotes the unit outside norm vector on $\partial B$. Using (4-7) we obtain

$$
\begin{aligned}
\left|\int_{B} v^{(p-2) / 2} \nabla \rho \cdot \nabla(v \cdot \nabla \rho)\right| & \leq C \varepsilon^{m-p}+\frac{1}{p}\left|\int_{B} v \cdot \nabla\left(v^{p / 2}\right)\right| \\
& \leq C \varepsilon^{m-p}+\frac{1}{p} \int_{\partial B} v^{p / 2} d \xi .
\end{aligned}
$$


Combining (4-3), (4-5), and (4-7) we also have

$$
\left|\frac{1}{\varepsilon^{p}} \int_{B}(1-\rho)(v \cdot \nabla \rho)\right| \leq \frac{1}{2 \varepsilon^{p}}\left|\int_{B}(1-\rho)^{2} \operatorname{div} v-\int_{\partial B}(1-\rho)^{2} d \xi\right| \leq C \varepsilon^{m-p} .
$$

Substituting this and (4-9) into (4-8) yields

$$
\left|\int_{\partial B} v^{(p-2) / 2}(v \cdot \nabla \rho)^{2} d \xi\right| \leq C \varepsilon^{m-p}+\frac{1}{p} \int_{\partial B} v^{p / 2} d \xi
$$

Applying (4-5), (4-3) and (4-10), we obtain for any $\delta \in(0,1)$,

$$
\begin{aligned}
& \int_{\partial B} v^{p / 2} d \xi=\int_{\partial B} v^{(p-2) / 2}\left(1+\sum_{i=1}^{n-1}\left(\tau_{i} \cdot \nabla \rho\right)^{2}+(v \cdot \nabla \rho)^{2}\right) d \xi \\
& \leq C(\delta) \varepsilon^{m-p}+(1 / p+2 \delta) \int_{\partial B} v^{p / 2} d \xi,
\end{aligned}
$$

where $\tau_{i}, i=1,2, \ldots, n-1$, denotes the unit tangent vector on $\partial B$ and $\tau_{i} \perp \tau_{j}$ when $i \neq j$. Choosing $\delta>0$ sufficiently small yields

$$
\int_{\partial B} v^{p / 2} d \xi \leq C \varepsilon^{m-p}
$$

Multiplying both sides of (4-4) by $(1-\rho)$ and integrating over $B$, we have

$$
\int_{B} v^{(p-2) / 2}|\nabla \rho|^{2}+\frac{1}{\varepsilon^{p}} \int_{B}(1-\rho)^{2}=-\int_{\partial B} v^{(p-2) / 2}(v \cdot \nabla \rho)(1-\rho) d \xi .
$$

Thus, applying Hölder's inequality, (4-3), (4-5), (4-6) and (4-11), we obtain

$$
E\left(\rho_{m}, B\right) \leq C \varepsilon^{(m-p)(p-1) / p}\left|\int_{\partial B}\left(1-\left|u_{\varepsilon}\right|\right)^{2} d \xi\right|^{1 / p} \leq C \varepsilon^{m-p+1} .
$$

Remark 4. Comparing (4-12) with (4-7), we see that the exponent of $\varepsilon$ in the upper bound of $E\left(\rho_{m}, B\right)$ is improved. We shall use $\rho_{m}$ as a comparison function to improve the exponent of $\varepsilon$ in the upper bound of $E_{\varepsilon}\left(u_{\varepsilon}, B\right)$.

Proposition 4.3. Set $h=\left|u_{\varepsilon}\right|$. Then for any $\delta \in(0,1 / 2)$, there is $C>0$ such that

$$
\begin{aligned}
\frac{1}{p} \int_{B}|\nabla h|^{p}+\frac{1}{4 \varepsilon^{p}} \int_{B}\left(1-h^{2}\right)^{2} & \leq C \varepsilon^{m-p+1}+\delta \int_{B}\left|\nabla u_{\varepsilon}\right|^{p} \\
& +C\left(\int_{B(x, 2 r)}\left|\nabla u_{\varepsilon}\right|^{p}+1\right)\left(\int_{B}\left(1-h^{2}\right)^{2}\right)^{t /(p+t)} .
\end{aligned}
$$

Here $t$ is the constant in Proposition 3.5.

Proof. Let $U_{\varepsilon}=\rho_{m} w$ on $B$ and $U_{\varepsilon}=u_{\varepsilon}$ on $B_{1} \backslash B$, where $w=u_{\varepsilon} /\left|u_{\varepsilon}\right|$. Since $u_{\varepsilon}$ is a minimizer of $E_{\varepsilon}(u, G)$, we have

$$
E_{\varepsilon}\left(u_{\varepsilon}, G\right) \leq E_{\varepsilon}\left(U_{\varepsilon}, B_{1}\right)=E_{\varepsilon}\left(\rho_{m} w, B\right)+E_{\varepsilon}\left(u_{\varepsilon}, B_{1} \backslash B\right) .
$$


This means $E_{\varepsilon}\left(u_{\varepsilon}, B\right) \leq E_{\varepsilon}\left(\rho_{m} w, B\right)$. Noting that

$$
\begin{aligned}
\int_{B}\left(\left|\nabla \rho_{m}\right|^{2}+\rho_{m}^{2}|\nabla w|^{2}\right)^{p / 2} d x-\int_{B}\left(\rho_{m}^{2}|\nabla w|^{2}\right)^{p / 2} d x= & \\
\frac{p}{2} \int_{B} \int_{0}^{1}\left(\left(\left|\nabla \rho_{m}\right|^{2}+\rho_{m}^{2}|\nabla w|^{2}\right)^{(p-2) / 2} s\right. & \left.+\left(\rho_{m}^{2}|\nabla w|^{2}\right)^{(p-2) / 2}(1-s)\right) d s\left|\nabla \rho_{m}\right|^{2} d x \\
& \leq C \int_{B}\left(\left|\nabla \rho_{m}\right|^{p}+\left|\nabla \rho_{m}\right|^{2}|\nabla w|^{p-2}\right) d x
\end{aligned}
$$

and using Hölder's inequality, (4-6), and (4-12), we have, for any $\delta \in(0,1)$,

$$
\begin{aligned}
& E_{\varepsilon}\left(u_{\varepsilon}, B\right) \leq E_{\varepsilon}\left(\rho_{m} w, B\right) \\
& \leq \frac{1}{p} \int_{B}\left(\rho_{m}^{2}|\nabla w|^{2}\right)^{p / 2}+C \int_{B}\left(\left|\nabla \rho_{m}\right|^{p}\right. \\
& \left.+\left|\nabla \rho_{m}\right|^{2}|\nabla w|^{p-2}\right)+\frac{1}{4 \varepsilon^{p}} \int_{B}\left(1-\rho_{m}^{2}\right)^{2} \\
& \leq \frac{1}{p} \int_{B}|\nabla w|^{p}+C \varepsilon^{m+1-p}+\delta \int_{B}\left|\nabla u_{\varepsilon}\right|^{p} .
\end{aligned}
$$

Combining this with Jensen's inequality we obtain

$$
\begin{aligned}
\frac{1}{p} \int_{B}|\nabla h|^{p}+\frac{1}{p} & \int_{B}\left(h^{p}-1\right)|\nabla w|^{p}+\frac{1}{4 \varepsilon^{p}} \int_{B}\left(1-h^{2}\right)^{2} \\
& \leq E_{\varepsilon}\left(u_{\varepsilon}, B\right)-\frac{1}{p} \int_{B}|\nabla w|^{p} \leq C \varepsilon^{m-p+1}+\delta \int_{B}\left|\nabla u_{\varepsilon}\right|^{p}
\end{aligned}
$$

In view of (3-10) and Proposition 3.5, we get

$$
\begin{aligned}
\frac{1}{p} \int_{B}\left(1-h^{p}\right)\left|\nabla w_{\varepsilon}\right|^{p} & \leq \frac{2^{p}}{p} \int_{B}\left(1-h^{p}\right) h^{p}\left|\nabla w_{\varepsilon}\right|^{p} \\
& \leq C(R)\left(\int_{B(x, 2 r)}\left|\nabla u_{\varepsilon}\right|^{p}+1\right)\left(\int_{B}\left(1-h^{2}\right)^{2}\right)^{t /(p+t)} .
\end{aligned}
$$

Substituting this into (4-13) yields

$$
\begin{aligned}
\frac{1}{p} \int_{B}|\nabla h|^{p}+\frac{1}{4 \varepsilon^{p}} & \int_{B}\left(1-h^{2}\right)^{2} \leq C \varepsilon^{m-p+1}+\delta \int_{B}\left|\nabla u_{\varepsilon}\right|^{p} \\
& +C\left(\int_{B(x, 2 r)}\left|\nabla u_{\varepsilon}\right|^{p}+1\right)\left(\int_{B}\left(1-h^{2}\right)^{2}\right)^{t /(p+t)} .
\end{aligned}
$$

Proof of Theorem 4.1. Step 1. Using (3-10) we may write $w=u_{\varepsilon} /\left|u_{\varepsilon}\right|$ on $B(x, 3 R)$. Substituting this into (2-1) yields that

$$
\int_{B(x, 3 R)}|\nabla u|^{p-2}(w \nabla h+h \nabla w) \nabla \psi=\frac{1}{\varepsilon^{p}} \int_{B(x, 3 R)} h w \psi\left(1-h^{2}\right)
$$


or $\operatorname{div}\left(|\nabla u|^{p-2}(w \nabla h+h \nabla w)\right)+1 / \varepsilon^{p} h w\left(1-h^{2}\right)=0$ in the distribution sense. Taking $\psi=w \zeta$ where $\zeta \in W_{0}^{1, p}(B(x, 3 R))$, and noting that $w \nabla w=\frac{1}{2} \nabla\left(|w|^{2}\right)=0$, we obtain

$$
\frac{1}{\varepsilon^{p}} \int_{B(x, 3 R)} h\left(1-h^{2}\right) \zeta=\int_{B(x, 3 R)}|\nabla u|^{p-2}\left(\nabla h \nabla \zeta+h|\nabla w|^{2} \zeta\right)
$$

In addition, we also have $\operatorname{div}\left(|\nabla u|^{p-2}(w \nabla h+h \nabla w)\right) \wedge w=0$ in the distribution sense. Together with $|w|=1$, this implies

$$
\int_{B(x, 3 R)}|\nabla u|^{p-2} h(w \wedge \nabla w) \nabla \zeta=0
$$

Using this with Theorem 6.1 (which will be proved in Section 6), we can deduce that

$$
\int_{B}|\nabla u|^{p-2} h^{2}|\nabla w|^{2} \leq C\left(\int_{B(x, 2 r)}|\nabla u|^{p}\right)^{1-2 / p} .
$$

Applying (4-17) and Hölder's inequality we have, for any $\delta \in(0,1)$,

$$
\text { (4-18) } \begin{aligned}
\int_{B}|\nabla u|^{p} & =\int_{B}|\nabla u|^{p-2}\left(h^{2}|\nabla w|^{2}+|\nabla h|^{2}\right) \\
& \leq C\left(\int_{B(x, 2 r)}|\nabla u|^{p}\right)^{1-2 / p}+\delta \int_{B}|\nabla u|^{p}+C(\delta)\left(\int_{B}|\nabla h|^{p}\right) .
\end{aligned}
$$

Substituting (4-15) into (4-18) and choosing $\delta>0$ sufficiently small we see that

(4-19) $\int_{B}|\nabla u|^{p} \leq C\left(\int_{B(x, 2 r)}|\nabla u|^{p}\right)^{1-2 / p}+C \varepsilon^{m-p+1}$

$$
+C\left(\int_{B(x, 2 r)}\left|\nabla u_{\varepsilon}\right|^{p}+1\right)\left(\int_{B}\left(1-h^{2}\right)^{2}\right)^{t /(p+t)} .
$$

From (4-2) it follows that $\int_{B(x, 2 r)}|\nabla u|^{p} \leq C \varepsilon^{m-p}$. Substituting this into (4-19) yields

(4-20) $\int_{B}|\nabla u|^{p} \leq C\left(\varepsilon^{m-p}\right)^{1-2 / p}+C \varepsilon^{m-p+1}+C \varepsilon^{m-p+m t /(p+t)}=: I_{1}+I_{2}+I_{3}$.

Step 2. If $m \leq p / 2$, then $m+1-p \leq(m-p)(1-2 / p)$. Now, $I_{1} \leq I_{2}$. Let $k_{0}$ be an integer such that $m+1 \leq(1+t /(p+t))^{k_{0}} m$. 
Assume $\zeta$ is in $C_{0}^{\infty}(B(x, 2 R),[0,1])$ and satisfies $|\nabla \zeta| \leq C$ and $\zeta=1$ on $B_{m+1 / 2}$. Taking the test function as $h \zeta(1-h)$ in (4-16), we have

$$
\begin{aligned}
\frac{1}{\varepsilon^{p}} \int_{B} h^{2}\left(1-h^{2}\right) \zeta(1-h)+\int_{B}|\nabla u|^{p-2}|\nabla h|^{2} h \zeta \\
=\int_{B}|\nabla u|^{p-2} \nabla h \nabla \zeta h(1-h)+\int_{B}|\nabla u|^{p} \zeta(1-h) \leq C \int_{B}|\nabla u|^{p} .
\end{aligned}
$$

Noting that $\zeta=1$ on $B_{m+1 / 2}$ and applying (4-20), we obtain

$$
\int_{B_{m+1 / 2}}\left(1-h^{2}\right)^{2} \leq C \varepsilon^{m(1+t /(p+t))} \quad \text { for } \varepsilon \in\left(0, \varepsilon_{0}\right) .
$$

On the other hand, as in the derivation of (4-13), for $B_{m+1 / 2}$ we rewrite Proposition 4.3 and still conclude that for any $\delta>0$,

$$
\begin{aligned}
\frac{1}{p} \int_{B_{m+1 / 2}}|\nabla h|^{p} & +\frac{1}{4 \varepsilon^{p}} \int_{B_{m+1 / 2}}\left(1-h^{2}\right)^{2} \\
& \leq C \varepsilon^{m-p+1}+\frac{1}{p} \int_{B_{m+1 / 2}}\left(1-h^{p}\right)|\nabla w|^{p}+\delta \int_{B_{m+1 / 2}}\left|\nabla u_{\varepsilon}\right|^{p}
\end{aligned}
$$

To estimate the second term of the right-hand side of (4-22), we apply (4-21) to obtain

$$
\frac{1}{p} \int_{B_{m+1 / 2}}\left(1-h^{p}\right)|\nabla w|^{p} \leq C \varepsilon^{\left(m+\frac{t}{p+t} m\right) \frac{t}{p+t}+m+\frac{t}{p+t} m-p}=C \varepsilon^{m(1+t /(p+t))^{2}-p}
$$

by a similar derivation to (4-14). Substituting this into (4-22) yields

$$
\frac{1}{p} \int_{B_{m+1 / 2}}|\nabla h|^{p} \leq C\left(\varepsilon^{m-p+1}+\varepsilon^{m(1+t /(p+t))^{2}-p}\right)+\delta \int_{B_{m+1 / 2}}\left|\nabla u_{\varepsilon}\right|^{p} .
$$

Using this instead of (4-15) and choosing $\delta>0$ sufficiently small we can improve (4-20) to

$$
\int_{B_{m+1 / 2}}\left|\nabla u_{\varepsilon}\right|^{p} \leq C+C\left(\varepsilon^{m-p+1}+\varepsilon^{m(1+t /(p+t))^{2}-p}\right) \leq C \varepsilon^{m(1+t /(p+t))^{2}-p} .
$$

We have improved the exponent $m(1+t /(p+t))-p$ of $\varepsilon$ to $m(1+t /(p+t))^{2}-p$, though the integral domain $B$ has shrunk to $B_{m+1 / 2}$. By induction, it can be derived in $k_{0}$ steps that

$$
\int_{B_{m+1-1 / 2^{k_{0}-1}}}\left|\nabla u_{\varepsilon}\right|^{p} \leq C+C\left(\varepsilon^{m-p+1}+\varepsilon^{m(1+t /(p+t))^{k_{0}}-p}\right) .
$$


Noting the definition of $k_{0}$, we obtain (4-2) for $j=m+1$ :

$$
\int_{B_{m+1}}\left|\nabla u_{\varepsilon}\right|^{p} \leq \int_{B_{m+1-1 / 2^{k} 0^{-1}}}\left|\nabla u_{\varepsilon}\right|^{p} \leq C\left(\varepsilon^{m-p+1}+1\right) .
$$

Step 3. If $m>p / 2$, then $(m-p)(1-2 / p)<m+1-p$. Let $k \geq 3$ be an integer such that $(m-p)(1-2 / p)^{k} \leq m+1-p<(m-p)(1-2 / p)^{k+1}$. Now (4-20) becomes

$$
\int_{B}|\nabla u|^{p} \leq C\left(\varepsilon^{m-p}\right)^{1-2 / p}+C \varepsilon^{m-p+m t /(p+t)} .
$$

Proceeding as in Step 2, we improve the exponent $m-p+m t /(p+t)$ of $\varepsilon$ to $(m-p)(1-2 / p)$, since we can find $k_{0} \in \mathbb{Z}$ such that $m(1+t /(p+t))^{k_{0}}-p$ is greater than $(m-p)(1-2 / p)$. At the same time, the integral domain $B(x, r)$ shrinks. Namely, there is a constant $r_{1} \in\left(R_{m+1}, r\right)$ such that

$$
\int_{B\left(x, r_{1}\right)}\left|\nabla u_{\varepsilon}\right|^{p} \leq C \varepsilon^{(m-p)(1-2 / p)} .
$$

Hence as in the derivation of (4-21),

$$
\int_{B(x, r)}\left(1-h^{2}\right)^{2} \leq C \varepsilon^{(m-p)(1-2 / p)+p} .
$$

Substituting these into (4-19) we have

$$
\begin{aligned}
& \int_{B\left(x, r_{1} / 2\right)}\left|\nabla u_{\varepsilon}\right|^{p} \\
& \quad \leq C \varepsilon^{m+1-p}+C\left(\int_{B(x, r)}\left|\nabla u_{\varepsilon}\right|^{p}\right)^{1-\frac{2}{p}}+C \int_{B(x, r)}\left|\nabla u_{\varepsilon}\right|^{p}\left(\int_{B(x, r)}\left(1-h^{2}\right)^{2}\right)^{\frac{t}{p+t}} \\
& \leq C \varepsilon^{m+1-p}+C \varepsilon^{(m-p)(1-2 / p)^{2}}+C \varepsilon^{(m-p)(1-2 / p)+((m-p)(1-2 / p)+p)(t /(p+t))} \\
& \leq C \varepsilon^{(m-p)(1-2 / p)^{2}}+C \varepsilon^{(m-p)(1-2 / p)+((m-p)(1-2 / p)+p) t /(p+t)}
\end{aligned}
$$

Again by an argument analogous to Step 2, we improve the exponent of $\varepsilon$ in the last term to $(m-p)(1-2 / p)^{2}$. Namely, there is a constant $r_{2} \in\left(R_{m+1}, r_{1} / 2\right)$ such that

$$
\int_{B\left(x, r_{2}\right)}\left|\nabla u_{\varepsilon}\right|^{p} \leq C \varepsilon^{(m-p)(1-2 / p)^{2}} .
$$

By induction, it follows that

$$
\int_{B\left(x, r_{k-1}\right)}\left|\nabla u_{\varepsilon}\right|^{p} \leq C \varepsilon^{(m-p)(1-2 / p)^{k}} .
$$


Combining this with (4-19), and noting the definition of $k$, we obtain

$$
\begin{aligned}
& \int_{B\left(x, r_{k-1} / 2\right)}\left|\nabla u_{\varepsilon}\right|^{p} \\
& \quad \leq C \varepsilon^{m+1-p}+C \varepsilon^{(m-p)(1-2 / p)^{k+1}}+C \varepsilon^{(m-p)(1-2 / p)^{k}+\left((m-p)(1-2 / p)^{k}+p\right)(t /(p+t))} \\
& \quad \leq C \varepsilon^{m+1-p}+C \varepsilon^{(m-p)(1-2 / p)^{k}+\left((m-p)(1-2 / p)^{k}+p\right)(t /(p+t))} .
\end{aligned}
$$

By the same discussion as in Step 2, we may also improve the exponent of $\varepsilon$ to $m+1-p$, and the integral domain shrinks. Namely, we have (4-2) with $j=m+1$ :

$$
\int_{B\left(x, r_{k-1} / 2\right)}\left|\nabla u_{\varepsilon}\right|^{p} \leq C \varepsilon^{m+1-p} .
$$

Theorem 4.4 (Uniform estimation). For any compact $K \subset B_{1} \backslash\left\{0, a_{1}, a_{2}, \ldots, a_{N}\right\}$, there exists a constant $C>0$ independent of $\varepsilon$ such that $E_{\varepsilon}\left(u_{\varepsilon}, K\right) \leq C$.

Proof. We only prove the theorem for the ball $B(x, R)$ in $B_{1} \backslash\left\{0, a_{1}, a_{2}, \ldots, a_{N}\right\}$. Theorem 4.1 shows that

$$
E_{\varepsilon}\left(u_{\varepsilon}, B_{[p]}\right) \leq C \varepsilon^{[p]-p} .
$$

The integral mean value theorem and (4-24) imply that there exists a constant $r \in\left[R_{[p]}, R_{[p]+1 / 2}\right]$ such that

$$
\int_{\partial B(x, r)}\left|\nabla u_{\varepsilon}\right|^{p} d \xi+\frac{1}{\varepsilon^{p}} \int_{\partial B(x, r)}\left(1-\left|u_{\varepsilon}\right|^{2}\right)^{2} d \xi \leq C \varepsilon^{[p]-p} .
$$

Consider the functional

$$
E(\rho, B)=\frac{1}{p} \int_{B}\left(|\nabla \rho|^{2}+1\right)^{p / 2}+\frac{1}{2 \varepsilon^{p}} \int_{B}(1-\rho)^{2},
$$

where $B=B(x, r)$. It is easy to see the existence of the minimizer $\rho_{[p]}$ of $E(\rho, B)$ on $W_{\left|u_{\varepsilon}\right|}^{1, p}\left(B, R^{+} \cup\{0\}\right)$. Similar to the proof of Proposition 4.2, from (4-24) and (4-25) we can deduce $E\left(\rho_{[p]}, B\right) \leq C \varepsilon^{[p]-p+1}$. Thus, for any $\delta \in(0,1)$,

$$
E_{\varepsilon}\left(u_{\varepsilon}, B\right) \leq E_{\varepsilon}\left(\rho_{[p]} w, B\right) \leq \frac{1}{p} \int_{B}|\nabla w|^{p}+C \varepsilon^{[p]+1-p}+\delta \int_{B}\left|\nabla u_{\varepsilon}\right|^{p} .
$$

As in the derivation of (4-8), it follows that

$$
\text { (4-26) } \begin{aligned}
\frac{1}{p} \int_{B}|\nabla h|^{p}+\frac{1}{4 \varepsilon^{p}} \int_{B} & \left(1-h^{2}\right)^{2} \\
& \leq C \varepsilon^{[p]+1-p}+\delta \int_{B}\left|\nabla u_{\varepsilon}\right|^{p}+\frac{1}{p} \int_{B}\left(1-h^{p}\right)|\nabla w|^{p} .
\end{aligned}
$$

To estimate the third term of the right-hand side, we shall do as in the proof of (4-14) and (4-15) and apply $\left(1 / \varepsilon^{p}\right) \int_{B}\left(1-h^{2}\right)^{2} \leq C \varepsilon^{[p]-p}$, which is implied by 
(4-24). As a result, there exists $t \in(0,1 / 2)$ such that

$$
\frac{1}{p} \int_{B}\left(1-h^{p}\right)|\nabla w|^{p} \leq C \varepsilon^{[p]+[p] t /(p+t)-p} .
$$

Substituting this into (4-26) yields

$$
\frac{1}{p} \int_{B}|\nabla h|^{p}+\frac{1}{4 \varepsilon^{p}} \int_{B}\left(1-h^{2}\right)^{2} \leq C\left(\varepsilon^{[p]+1-p}+\varepsilon^{[p]+[p] t /(p+t)-p}\right)+\delta \int_{B}\left|\nabla u_{\varepsilon}\right|^{p} .
$$

Combining this with (4-18) and choosing $\delta$ sufficiently small, we obtain

$$
\int_{B}\left|\nabla u_{\varepsilon}\right|^{p} \leq C \varepsilon^{[p]-p+1}+C \varepsilon^{[p]-p+t m /(p+t)}+C \varepsilon^{([p]-p)(1-2 / p)}+C .
$$

By a same argument of Steps 2 and 3, we may improve the exponents of $\varepsilon$ in the second and the third terms of the right hand side to $[p]-p+1$. Thus, for some shrinking domain $B_{[p]+1} \subset B$, there exists $C>0$ independent of $\varepsilon \in\left(0, \varepsilon_{0}\right)$, such that

$$
\int_{B_{[p]+1}}\left|\nabla u_{\varepsilon}\right|^{p} \leq C+C \varepsilon^{[p]+1-p} \leq C .
$$

\section{Convergence}

There may be several minimizers of $E_{\varepsilon}\left(u, B_{1}\right)$. One of them, denoted by $\tilde{u}_{\varepsilon}$, can be obtained as the limit of a subsequence $u_{\varepsilon}^{\tau_{k}}$ of the minimizers $u_{\varepsilon}^{\tau}$ of the regularized functionals

$$
E_{\varepsilon}^{\tau}(u, G)=\frac{1}{p} \int_{G}\left(|\nabla u|^{2}+\tau\right)^{p / 2} d x+\frac{1}{4 \varepsilon^{p}} \int_{G}\left(1-|u|^{2}\right)^{2} d x, \quad \text { for } \tau \in(0,1)
$$

in $W_{g}^{1, p}\left(B_{1}, R^{n}\right)$ as $\tau_{k} \rightarrow 0$, namely

$$
\lim _{\tau_{k} \rightarrow 0} u_{\varepsilon}^{\tau_{k}}=\tilde{u}_{\varepsilon} \text { in } W^{1, p}\left(B_{1}, R^{n}\right) .
$$

We call $\tilde{u}_{\varepsilon}$ the regularized minimizer of $E_{\varepsilon}\left(u, B_{1}\right)$. For the regularized minimizer we shall establish the $C^{1, \alpha}$ convergence when $p>n-t$ and $p \neq n$.

It is not difficult to see that the minimizer $u_{\varepsilon}^{\tau}$ of $E_{\varepsilon}^{\tau}\left(u, B_{1}\right)$ solves

$$
-\operatorname{div}\left(\left(|\nabla u|^{2}+\tau\right)^{p-2} \nabla u\right)=\frac{1}{\varepsilon^{p}} u\left(1-|u|^{2}\right) \quad \text { on } B_{1}
$$

and satisfies $\left|u_{\varepsilon}^{\tau}\right| \leq 1$ on $\bar{B}_{1}$. As (3-10) and Theorem 1.2 hold for $u_{\varepsilon}^{\tau}$, the following results are also true: for any compact subset $K$ of $B_{1} \backslash\left\{0, a_{1}, a_{2}, \ldots, a_{N}\right\}$, there is $C>0$ such that

$$
\left|u_{\varepsilon}^{\tau}(x)\right| \geq 1 / 2 \text { for all } x \in K
$$


and

$$
E_{\varepsilon}^{\tau}\left(u_{\varepsilon}^{\tau}, K\right) \leq C .
$$

Proposition 5.1. Assume $p>n-t$ and $p \neq n$, where $t$ is the constant in Proposition 3.5. Then for any compact subset $K \subset B_{1} \backslash\left\{0, a_{1}, a_{2}, \ldots, a_{N}\right\}$ and arbitrary $l>1$, there exists a constant $C>0$ which is independent of $\varepsilon, \tau$, such that

$$
\left\|\nabla u_{\varepsilon}^{\tau}\right\|_{L^{l}\left(K, R^{n}\right)} \leq C=C(K, l) .
$$

Proof. Step 1. Write $v=|\nabla u|^{2}+\tau$ in (5-2). Differentiating (5-2) with respect to $x_{j}$, we obtain

$$
-\left(v^{(p-2) / 2} u_{x_{i}}\right)_{x_{i} x_{j}}=\frac{1}{\varepsilon^{p}}\left(u\left(1-|u|^{2}\right)\right)_{x_{j}} .
$$

Take $R>0$ so that $B=B(\cdot, 3 R) \Subset B_{1} \backslash\left\{0, a_{1}, a_{2}, \ldots, a_{N}\right\}$. Let $\zeta \in C_{0}^{\infty}(B,[0,1])$ be a function such that $\zeta=1$ on $B(\cdot, R)$ and $\zeta=0$ on $B(\cdot, 3 R) \backslash B(\cdot, 2 R)$, with $|\nabla \zeta| \leq C$ on $B(\cdot, 3 R)$. Now integrate over $B(\cdot, 3 R)$ the inner product of the both sides of (5-6) with $u_{x_{j}} v^{b} \zeta^{2}(b \geq 0)$ to obtain

$$
\int_{B}\left(v^{(p-2) / 2} u_{x_{i}}\right)_{x_{j}}\left(\zeta^{2} u_{x_{j}}\right)_{x_{i}}=\frac{1}{\varepsilon^{p}} \int_{B}\left(1-|u|^{2}\right) \zeta^{2}\left(u_{x_{j}}\right)^{2} v^{b}-\frac{1}{2 \varepsilon^{p}} \int_{B} \zeta^{2}\left(\left(|u|^{2}\right)_{x_{j}}\right)^{2} v^{b} .
$$

Summing over $j=1,2, \ldots, n$ and computing the term of the left hand side yields

$$
\begin{aligned}
\int_{B} \zeta^{2} v^{(p+2 b-2) / 2} \sum_{j=1}^{n}\left|\nabla u_{x_{j}}\right|^{2}+ & \frac{p+2 b-2}{4} \int_{B} \zeta^{2} v^{(p+2 b-4) / 2}|\nabla v|^{2} \\
& +\frac{b(p-2)}{2} \int_{B} v^{(p+2 b-6) / 2}(\nabla u \cdot \nabla v)^{2} \\
\leq \frac{1}{\varepsilon^{p}} \int_{B}\left(1-|u|^{2}\right) \zeta^{2} v^{b+1}+2 \mid & \sum_{j=1}^{n} \int_{B}\left(v^{(p-2) / 2} \nabla u\right)_{x_{j}} u_{x_{j}} v^{b} \zeta \nabla \zeta \mid .
\end{aligned}
$$

From (5-2) and (5-3), it follows that

$$
\frac{1}{\varepsilon^{p}}\left(1-|u|^{2}\right)=\frac{-u}{|u|^{2}} \operatorname{div}\left(v^{(p-2) / 2} \nabla u\right) .
$$

Applying Young's inequality, we derive that for any $\delta \in(0,1)$,

$$
\begin{aligned}
& \frac{1}{\varepsilon^{p}} \int_{B}\left(1-|u|^{2}\right) \zeta^{2} v^{b+1} \leq C(\delta) \int_{B} \zeta^{2} v^{(p+2 b+2) / 2} \\
& \quad+\delta \int_{B} \zeta^{2} v^{(p+2 b-4) / 2}|\nabla v|^{2}+\delta \int_{B} \zeta^{2} \sum_{j=1}^{n}\left|\nabla u_{x_{j}}\right|^{2} v^{(p+2 b-2) / 2}
\end{aligned}
$$


where $\varepsilon, \tau \in\left(0, \varepsilon_{0}\right)$. Using the Young inequality again, for any $\delta \in(0,1)$

$$
\begin{aligned}
(5-10) & \left|\sum_{j=1}^{n} \int_{B}\left(v^{(p-2) / 2} \nabla u\right)_{x_{j}} u_{x_{j}} v^{b} \zeta \nabla \zeta\right| \\
& \leq \delta \int_{B} v^{(p+2 b-4) / 2}|\nabla v|^{2} \zeta^{2}+C(\delta) \int_{B} v^{(p+2 b) / 2}|\nabla \zeta|^{2}
\end{aligned}
$$

Substituting (5-9)- (5-10) into (5-7) and choosing $\delta$ small enough yields

$$
\begin{gathered}
\int_{B} \zeta^{2} v^{(p+2 b-2) / 2} \sum_{j=1}^{n}\left|\nabla u_{x_{j}}\right|^{2}+\frac{p+2 b-2}{4} \int_{B} \zeta^{2} v^{(p+2 b-4) / 2}|\nabla v|^{2} \\
+\frac{b(p-2)}{2} \int_{B} v^{(p+2 b-6) / 2}(\nabla u \cdot \nabla v)^{2} \\
\leq C \int_{B} \zeta^{2} v^{(p+2 b+2) / 2}+C \int_{B} v^{(p+2 b) / 2}|\nabla \zeta|^{2}
\end{gathered}
$$

Step 2. When $p>2$, all the terms of the left-hand side of (5-11) are nonnegative. When $1<p<2$, first observe that

$$
v^{(p+2 b-2) / 2} \sum_{j=1}^{n}\left|\nabla u_{x_{j}}\right|^{2} \geq \frac{1}{4} v^{(p+2 b-4) / 2}|\nabla v|^{2} .
$$

Next, the third term of the left-hand side of (5-11) is not positive. However,

$$
\frac{b(p-2)}{2} \int_{B} \zeta^{2} v^{(p+2 b-6) / 2}(\nabla u \cdot \nabla v)^{2} \geq \frac{b(p-2)}{2} \int_{B} \zeta^{2} v^{(p+2 b-4) / 2}|\nabla v|^{2} .
$$

Hence, we can derive from (5-11) that

$$
\int_{B} \zeta^{2} v^{(p+2 b-4) / 2}|\nabla v|^{2} \leq C \int_{B} \zeta^{2} v^{(p+2 b+2) / 2}+C \int_{B} v^{(p+2 b) / 2}|\nabla \zeta|^{2} .
$$

To estimate $\int_{B} \zeta^{2} v^{(p+2 b+2) / 2}$, we take $\phi=\zeta^{2 / q} v^{(p+2 b+2) / 2 q}$ in the interpolation inequality

$$
\|\phi\|_{L^{q}} \leq C\|\nabla \phi\|_{L^{\kappa}}^{\alpha}\|\phi\|_{L^{1}}^{1-\alpha}, \quad q \in(1, n \kappa /(n-\kappa)),
$$

where

$$
\alpha=\left(1-\frac{1}{q}\right)\left(1-\frac{n-\kappa}{n \kappa}\right)^{-1} \in(0,1)
$$


Thus,

$$
\text { (5-14) } \begin{aligned}
\int_{B} & \zeta^{2} v^{\frac{p+2 b+2}{2}} \\
& \leq C\left(\int_{B} \zeta^{\frac{2}{q}} v^{\frac{p+2 b+2}{2 q}}\right)^{q(1-\alpha)} \times \\
& \left(\int_{B} \zeta^{\kappa\left(\frac{2}{q}-1\right)}|\nabla \zeta|^{\kappa} v^{\kappa \frac{p+2 b+2}{2 q}}+\frac{p+2 b+2}{2 q}\left(\int_{B} \zeta^{2} v^{\frac{p+2 b-4}{2}}|\nabla v|^{2}\right)^{\frac{\kappa}{2}}\right. \\
& \left.\times\left(\int_{B} \zeta^{\frac{2 \kappa}{2-\kappa}\left(\frac{2}{q}-1\right)} v^{\frac{2 \kappa}{2-\kappa}\left(\frac{p+2 b+2}{2 q}-\frac{p+2 b}{4}\right)}\right)^{1-\frac{\kappa}{2}}\right)^{\frac{q \alpha}{\kappa}} .
\end{aligned}
$$

Step 3. Since $p>n-t$ and $p \neq n$, we can choose $\kappa$ such that $1<\kappa<2$ and $\kappa \in(2 n(2-t) /(2(p+2 b+2)-n t), 2 n /(n+2))$. Using $\kappa$, fix $q$ in the interval $(2 t(p+2 b+2) /(2(p+2 b+t)-\kappa t), n \kappa /(n-\kappa)) \subset(1,2)$. Thus, $q \alpha / 2<1$ and

(5-15) $\frac{p+2 b+2}{2 q}, \frac{\kappa(p+2 b+2)}{2 q}, \frac{2 \kappa}{2-\kappa}\left(\frac{p+2 b+2}{2 q}-\frac{p+2 b}{4}\right) \leq \frac{p+2 b+t}{2}$.

Let $b=0$. From Hölder's inequality, Proposition 3.5 and (5-4) it follows that

$$
\begin{aligned}
\int_{B} \zeta^{2 / q} v^{(p+2) / 2 q}+\int_{B} \zeta^{\kappa(2 / q-1)}|\nabla \zeta|^{\kappa} v^{\kappa(p+2) /(2 q)} & \leq C\left(\int_{B} v^{(p+t) / 2}\right)^{(p+2) /((p+t) q)} \\
& \leq C\left(\int_{B} v^{p / 2}\right)^{(p+2) /(p q)} \leq C .
\end{aligned}
$$

Substituting this into (5-14), and again using Hölder's inequality, Proposition 3.5 and (5-4), we obtain that for any $\delta \in(0,1)$,

$\int_{B} \zeta^{2} v^{(p+2) / 2} \leq C+C\left(\int_{B} \zeta^{2} v^{(p-4) / 2}|\nabla v|^{2}\right)^{q \alpha / 2} \leq C(\delta)+\delta \int_{B} \zeta^{2} v^{(p-4) / 2}|\nabla v|^{2}$,

since $q \alpha / 2<1$. Substituting this into (5-12), we see that $\int_{B} \zeta^{2} v^{(p-4) / 2}|\nabla v|^{2} \leq C$ or $\int_{B} \zeta^{2}|\nabla w|^{2} \leq C$, where $w=v^{p / 4}$. Since (5-4) implies $\int_{B} \zeta^{2}|w|^{2} \leq C$, we have $\|\zeta w\|_{H^{1}(B, R)}^{2} \leq C$, and thus the embedding inequality implies (5-5) when $n=2$. If $n \geq 3$, the embedding inequality gives

$$
\left(\int_{B}(\zeta w)^{r}\right)^{1 / r} \leq C\|\zeta w\|_{H^{1}(B, R)} \leq C,
$$

where $r \leq 2 n / n-2$. Now we set $G_{i}=B\left(x_{0}, R+R / 2^{i}\right)$. Take $\zeta$ such that $\zeta=1$ on $G_{1}$ and $\zeta=0$ on $B\left(x_{0}, 3 R\right) \backslash B\left(x_{0}, 2 R\right)$. Noting that $p>n-t$ and $t<4 / n$, choose $r=2+8 /(n p)$ in (5-16). Since $\zeta=1$ on $G_{1}$, we see that $\nabla u \in L^{s_{1}}\left(G_{1}\right)$ 
where $s_{1}=p+4 / n$, and

$$
\int_{G_{1}}|\nabla u|^{s_{1}} \leq C
$$

Step 4. To prove (5-5), we will choose $b>0$ and proceed in the same way as in Steps 1,2 and 3. However, Proposition 3.5 can not be applied, since it is only a result on the regularized functional $\int_{B_{1}} v^{p / 2} d x$ and is not valid on $\int_{B_{1}} v^{s / 2} d x$ for $s>p$. On the other hand, if we take $b \geq 2 / n$ from now on, the inequalities $p>n-t$ and $t<4 / n$ imply that $\kappa$ can be taken in $(2(2-t) n /(2 p+4 b+4-n t), 2 n /(n+2))$ with $t=0$. In view of this, suppose $t=0$ in the following calculation when proceeding as in Step 3.

Write $w=v^{(p+2 b) / 4}$. Set $b=2 / n$ and take $\zeta=1$ on $G_{2}$ and $\zeta=0$ on $B_{1} \backslash G_{1}$. Then from (5-17),

$$
\int_{G_{1}} w^{2}=\int_{G_{1}} v^{(p+4 / n) / 2}=\int_{G_{1}}|\nabla u|^{s_{1}} \leq C .
$$

Noting (5-15) with $t=0$, we use Hölder's inequality to estimate the terms of the right-hand side of (5-14). Combining with (5-12), we have, for any $\delta \in(0,1)$,

$$
\int_{G_{1}} \zeta^{2} v^{(p+2 b-4) / 2}|\nabla v|^{2} \leq C(\delta)\left(1+\int_{G_{1}} w^{2}\right)^{\lambda}+\delta \int_{G_{1}} \zeta^{2} v^{(p+2 b-4) / 2}|\nabla v|^{2},
$$

where $\lambda>0$ only depends on $n, p$ and $b$. Choosing $\delta$ sufficiently small, we obtain $\|\zeta w\|_{H^{1}\left(G_{1}\right)}^{2} \leq C\left(1+\int_{G_{1}} w^{2}\right)^{\lambda} \leq C$. Applying the embedding theorem to $\zeta w$ and using that $\zeta=1$ on $G_{2}$, we obtain

$$
\int_{G_{2}}|\nabla u|^{s_{2}} \leq C
$$

where $s_{2}=s_{1}+4(n+2) / n^{2}=p+4 / n+4(n+2) / n^{2}=p+8 / n+8 / n^{2}$.

Step 5. Reset $b$ and $\zeta$ again. Applying (5-18) and following the same logic as Step 4 , we can improve $s_{2}$ to $s_{3}>s_{2}$. For any $l>1$, proceeding inductively, we may at last find $s_{i}$ for some $i$ such that $s_{i}>l$ and

$$
\int_{G_{i}}|\nabla u|^{s_{i}} \leq C
$$

where $G_{i} \subset B_{R}$. Thus (5-5) is proved.

We can extend Proposition 5.1 by means of Moser iteration.

Proposition 5.2. Assume $p>n-t$ and $p \neq n$. Then for any compact subset $K \subset B_{1} \backslash\left\{0, a_{1}, a_{2}, \ldots, a_{N}\right\}$, there exists a constant $C=C(K)>0$ independent of $\varepsilon, \tau$, such that

$$
\left\|\nabla u_{\varepsilon}^{\tau}\right\|_{L^{\infty}\left(K, R^{n}\right)} \leq C .
$$


Proof. Given any $x_{0} \in B(\cdot, 3 R) \subset B_{1} \backslash\left\{0, a_{1}, a_{2}, \ldots, a_{N}\right\}$, let $r>0$ be small such that $B\left(x_{0}, 2 r\right) \subset B(\cdot, 2 R)$. Denote $Q_{m}=B\left(x_{0}, r_{m}\right)$ where $r_{m}=r+r / 2^{m}$. Choose $\zeta_{m} \in C_{0}^{\infty}\left(Q_{m}, R\right)$ such that $\zeta_{m}=1$ on $Q_{m+1}$ and $\left|\nabla \zeta_{m}\right| \leq C r^{-1} 2^{m}, m=1,2, \ldots$ Integrate over $Q_{m}$ the inner product of the both sides of (5-6) with $\zeta_{m}^{2} v^{b} u_{x_{j}}, b \geq 1$. Then, as in the derivation of (5-12), we see that

(5-19) $\int_{Q_{m}} \zeta_{m}^{2} v^{(p+2 b-4) / 2}|\nabla v|^{2} \leq C \int_{Q_{m}} v^{(p+2 b) / 2}\left|\nabla \zeta_{m}\right|^{2}+C \int_{Q_{m}} \zeta_{m}^{2} v^{(p+2 b+2) / 2}$.

To estimate $\int_{Q_{m}} \zeta_{m}^{2} v^{(p+2 b+2) / 2}$, we take $\phi=\zeta_{m}^{2 / q} v^{(p+2 b+2) / 2 q}$ in the interpolation inequality (5-13). We then obtain

$$
\begin{aligned}
\int_{Q_{m}} \zeta_{m}^{2} v^{(p+2 b+2) / 2} \leq C\left(\int_{Q_{m}} \zeta_{m}^{2 / q} v^{(p+2 b+2) /(2 q)}\right)^{q(1-\alpha)} \\
\quad \times\left(\left(\frac{2}{q}\right)^{\kappa} \int_{Q_{m}} \zeta_{m}^{\kappa(2 / q-1)}\left|\nabla \zeta_{m}\right|^{\kappa} v^{\kappa(p+2 b+2) /(2 q)}\right. \\
\left.\quad+\left(\frac{p+2 b+2}{2 q}\right)^{\kappa} \int_{Q_{m}} \zeta_{m}^{2 \kappa / q} v^{\kappa((p+2 b+2) /(2 q)-1)}|\nabla v|^{\kappa}\right)^{q \alpha / \kappa}
\end{aligned}
$$

Now, we estimate the right-hand side. Choose $r \in(0,1)$ sufficiently small such that $\left|Q_{m}\right| \leq 1$. Take $\kappa \in(2 n /(p+4), 2 n /(n+2)) \cap(1,2)$; hence, $q$ can be chosen in $((\kappa(p+4)) /(p+2), n \kappa /(n-\kappa))$. This implies that (5-15) with $t=0$ is also true since $b \geq 1$. By using Hölder's inequality, we have

$$
\begin{aligned}
\int_{Q_{m}} \zeta_{m}^{2 / q} v^{(p+2 b+2) / 2 q} & \leq\left(\int_{Q_{m}} v^{(p+2 b) / 2}\right)^{(p+2 b+2) /(q(p+2 b))}, \\
\int_{Q_{m}} \zeta_{m}^{\kappa(2 / q-1)}\left|\nabla \zeta_{m}\right|^{\kappa} v^{\kappa(p+2 b+2) /(2 q)} & \leq \frac{2^{2 m}}{r^{\kappa}}\left(\int_{Q_{m}} v^{(p+2 b) / 2}\right)^{\kappa(p+2 b+2) /(q(p+2 b))},
\end{aligned}
$$

and

$$
\begin{aligned}
\int_{Q_{m}} \zeta_{m}^{(2 \kappa) / q} v^{\kappa((p+2 b+2) /(2 q)-1)}|\nabla v|^{\kappa} & \\
& \leq\left(\int_{Q_{m}} \zeta_{m}^{2} v^{(p+2 b-4) / 2}|\nabla v|^{2}\right)^{\kappa / 2}\left(\int_{Q_{m}} v^{(p+2 b) / 2}\right)^{\kappa((p+2 b+2) /(q(p+2 b))-1 / 2)} .
\end{aligned}
$$

Combining these inequalities with (5-19) and (5-20) yields

(5-21) $\quad I_{1} \leq C\left(\left(\frac{2^{m}}{r}\right)^{2} I_{2}+\left(\frac{4^{m}}{r}\right)^{q \alpha} I_{2}^{1+2 /(p+2 b)}\right.$

$$
\left.+\left(\frac{p+2 b+2}{2 q}\right)^{q \alpha} I_{1}^{q \alpha / 2} I_{2}^{1+2 /(p+2 b)-q \alpha / 2}\right)
$$


where

$$
I_{1}=\int_{Q_{m}} \zeta_{m}^{2} v^{(p+2 b-4) / 2}|\nabla v|^{2} \quad \text { and } \quad I_{2}=\int_{Q_{m}} v^{(p+2 b) / 2} .
$$

Let $p+2 b=s^{m}$ and $w=v^{(p+2 b) / 4}=v^{s^{m} / 4}$, with $s>1$ to be determined later. Using the Young inequality to treat the last term on the right side of (5-21), we obtain

$$
\begin{aligned}
\left(\frac{s^{m}+2}{2 q}\right)^{q \alpha} I_{1}^{q \alpha / 2} I_{2}^{1+2 /\left(s^{m}\right)}-q \alpha / 2 & \\
& \leq \delta I_{1}+C(\delta)\left(\frac{s^{m}+2}{2 q}\right)^{2 q \alpha /(2-q \alpha)} I_{2}^{2\left(1+2 /\left(s^{m}\right)-q \alpha / 2\right) /(2-q \alpha)} .
\end{aligned}
$$

Substituting this into (5-21), we get

$$
\begin{aligned}
\text { (5-22) } I_{1} \leq C(\delta)\left(\left(\frac{2^{m}}{r}\right)^{2} I_{2}+\left(\frac{4^{m}}{r^{t}}\right)^{q \alpha} I_{2}^{1+2 / s^{m}}\right. \\
\left.+\left(\frac{s^{m}+2}{2 q}\right)^{2 q \alpha /(2-q \alpha)} I_{2}^{2\left(1+2 / s^{m}-q \alpha / 2\right) /(2-q \alpha)}\right) .
\end{aligned}
$$

By the embedding theorem, for any $s \in(1, n /(n-2)]$,

$$
\begin{aligned}
\int_{Q_{m}}\left(\zeta_{m} w\right)^{2 s} \leq C(s)\left(\int_{Q_{m}}\left(\zeta_{m} w\right)^{2}+\int_{Q_{m}}\left|\nabla\left(\zeta_{m} w\right)\right|^{2}\right)^{s} & \\
& \leq C\left(\left(1+\left(\frac{2^{m}}{r}\right)^{2}\right) I_{2}+\left(\frac{s^{m}}{4}\right)^{2} I_{1}\right)^{s} .
\end{aligned}
$$

Combining this with (5-22) yields

$$
\begin{aligned}
\int_{Q_{m}}\left(\zeta_{m} w\right)^{2 s} \leq C(s, r, q, \kappa)( & \left(1+4^{m}+s^{2 m} 4^{m}\right) I_{2}+s^{2 m} 4^{q \alpha m} I_{2}^{1+2 / s^{m}} \\
& \left.+s^{2 m} s^{2 q \alpha m /(2-q \alpha)} I_{2}^{\left(1+2 / s^{m}-q \alpha / 2\right) 2 /(2-q \alpha)}\right)^{s} .
\end{aligned}
$$

If there is a subsequence of positive integers $\left\{m_{i}\right\}$ tending to infinity such that

$$
I_{2}=\int_{Q_{m_{i}}} v^{s^{m_{i}} / 2}<1,
$$

then letting $m_{i} \rightarrow \infty$ immediately yields

$$
\|v\|_{L^{\infty}\left(Q_{\infty}, R\right)} \leq C(r) .
$$

Otherwise, there must be a positive integer $m_{0}$ such that

$$
I_{2}=\int_{Q_{m}} v^{s^{m} / 2} \geq 1 \quad \text { for } m \geq m_{0}
$$

Since $\left(1+2 / s^{m}-q \alpha / 2\right)(2 /(2-q \alpha))=1+\left(2 / s^{m}\right)(2 /(2-q \alpha))>1$, the exponent of the last term in (5-23) is higher than those of the other terms. Now we compare 
the coefficients of the terms in (5-23). If we choose $s \in\left(1, \min \left\{n /(n-2), 2^{2-q \alpha}\right\}\right)$, then $4^{m}$ and $s^{2 q \alpha m /(2-q \alpha)}$ are less than $4^{(1+q \alpha) m}$. Thus,

$$
\int_{Q_{m}}\left(\zeta_{m} w\right)^{2 s} \leq C\left(\left(s^{2} 4^{1+q \alpha}\right)^{m} I_{2}^{1+\left(2 / s^{m}\right)(2 /(2-q \alpha))}\right)^{s} .
$$

This means

$$
\int_{Q_{m+1}} v^{s^{m+1} / 2} \leq\left(C_{0} C_{1}^{m}\right)^{s}\left(\int_{Q_{m}} v^{s^{m} / 2}\right)^{\left(1+C_{2} / s^{m}\right) s},
$$

where $C_{1}=\left(s^{2} 4^{1+q \alpha}\right)^{s}, C_{2}=4 /(2-q \alpha)$, and $C_{0}$ is a positive constant. Using the iteration lemma [Lei 2004, Proposition 2.3] and Proposition 5.1, we also obtain the estimate (5-24), completing the proof of Proposition 5.2.

Proposition 5.3. Assume $p>n-t$ and $p \neq n$. Suppose $\tilde{u}_{\varepsilon}$ is a regularized minimizer. Then for any compact subset $K \subset B_{1} \backslash\left\{0, a_{1}, a_{2}, \ldots, a_{N}\right\}$, there exists a constant $C=C(K)>0$ which is independent of $\varepsilon$ such that

$$
\left\|\left(1 / \varepsilon^{p}\right)\left(1-\left|\tilde{u}_{\varepsilon}\right|^{2}\right)\right\|_{L^{\infty}(K)} \leq C .
$$

Proof. Assume $B=B_{R} \Subset B_{1} \backslash\left\{0, a_{1}, a_{2}, \ldots, a_{N}\right\}$. Consider the inner product of the both sides of (5-2) with $u=u_{\varepsilon}^{\tau}$,

$$
-\operatorname{div}\left(v^{(p-2) / 2} \nabla u\right) u=\left(1 / \varepsilon^{p}\right)|u|^{2}\left(1-|u|^{2}\right)=|u|^{2} \psi,
$$

where $\psi=\psi_{\varepsilon}^{\tau}=\left(1 / \varepsilon^{p}\right)\left(1-\left|u_{\varepsilon}^{\tau}\right|^{2}\right)$. Combining this and $\nabla \psi=-\left(2 / \varepsilon^{p}\right) u \cdot \nabla u$ with the equality $-\operatorname{div}\left(v^{(p-2) / 2} \nabla u\right) u=-\operatorname{div}\left(v^{(p-2) / 2} u \cdot \nabla u\right)+v^{(p-2) / 2}|\nabla u|^{2}$, and noting (5-3), we then obtain

$$
(1 / 4) \psi \leq v^{(p-2) / 2}|\nabla u|^{2}+\left(\varepsilon^{p} / 2\right) \operatorname{div}\left(v^{(p-2) / 2} \nabla \psi\right) \quad \text { on } B .
$$

At the point $x_{0}$ where $\psi$ achieves its maximum on $B$, we have $\nabla \psi\left(x_{0}\right)=0$ and $\Delta \psi\left(x_{0}\right) \leq 0$. Hence at $x_{0}$,

$$
\operatorname{div}\left(v^{(p-2) / 2} \nabla \psi\right)=v^{(p-2) / 2} \Delta \psi+((p-2) / 2) v^{(p-4) / 2} \nabla v \nabla \psi \leq 0 .
$$

Combining this with (5-26) and using Proposition 5.2, we derive that

$$
\left\|\left(1 / \varepsilon^{p}\right)\left(1-\left|u_{\varepsilon}^{\tau}\right|^{2}\right)\right\|_{L^{\infty}(B)} \leq \psi\left(x_{0}\right) \leq C .
$$

When $p>n$, from (5-1) it follows that

$$
\lim _{\tau_{k} \rightarrow 0} u_{\varepsilon}^{\tau_{k}}=\tilde{u}_{\varepsilon} \quad \text { in } C\left(\bar{B}_{1}\right) .
$$

When $p \in(n-t, n)$, Proposition 3.5 still holds for $u_{\varepsilon}^{\tau}$. As in the proof of Proposition 3.4 , combining (5-1) with the embedding theorem we deduce (5-28). Letting $\tau \rightarrow 0$ in (5-27) and using (5-28), we reach (5-25) by a finite-covering argument. 
Proof of Theorem 1.3. According to Proposition 5.3, the right-hand side of the Euler-Lagrange equation

$$
-\operatorname{div}\left(|\nabla u|^{p-2} \nabla u\right)=\left(1 / \varepsilon^{p}\right) u\left(1-|u|^{2}\right)
$$

satisfied by $\tilde{u}$ is bounded on every compact subset $K \subset B_{1} \backslash\left\{0, a_{1}, a_{2}, \ldots, a_{N}\right\}$. Thus Tolksdorf's theorem [1983, p. 244, lines 19-23] yields that $\left\|\tilde{u}_{\varepsilon}\right\|_{C^{1, \beta}(K)} \leq C=$ $C(K)$ for some $\beta \in(0,1)$, where the constant $C$ does not depend on $\varepsilon$. Letting $\varepsilon \rightarrow 0$, we find a subsequence $\tilde{u}_{k}$ of $\tilde{u}_{\varepsilon}$ and a map $u_{*}$ such that $\tilde{u}_{\varepsilon} \rightarrow u_{*}$ in $C^{1, \alpha}(K)$ for all $\alpha \in(0, \beta)$. In addition, Remark 2 implies $u_{*}=u_{p}$, completing the proof.

\section{Proof of $(4-17)$}

Theorem 6.1. Assume $h=|u| \geq 1 / 2$ and let $w=u|u|^{-1}$. If $u \in W^{1, p}\left(B(x, 3 R), R^{n}\right)$ satisfies

$$
\int_{B(x, 3 R)}|\nabla u|^{p-2} h(w \wedge \nabla w) \nabla \zeta=0 \quad \text { for all } \zeta \in W_{0}^{1, p}(B(x, 3 R)),
$$

then for any $\rho \in(0,3 R / 2)$, there is $C>0$ such that

$$
\int_{B(x, \rho)}|\nabla u|^{p-2} h^{2}|\nabla w|^{2} \leq C\left(\int_{B(x, 2 \rho)}|\nabla u|^{p}\right)^{1-2 / p} .
$$

Proof. Let $\left\{e_{i}\right\}_{i=1}^{n}$ be an orthogonal basis of $R^{n}$. Since $|w|=1$ over $B(x, 3 R)$, we have the formula in $n$-dimension ball coordinates

$w=\cos \theta_{1} e_{1}+\sin \theta_{1} \cos \theta_{2} e_{2}+\sin \theta_{1} \sin \theta_{2} \cos \theta_{3} e_{3}+\ldots$

$$
+\sin \theta_{1} \ldots \sin \theta_{n-2} \cos \theta_{n-1} e_{n-1}+\sin \theta_{1} \ldots \sin \theta_{n-2} \sin \theta_{n-1} e_{n} .
$$

As $h \geq 1 / 2$, there is no zero of $u$ in $B(x, 3 R)$. This implies $\operatorname{deg}(w, \partial \Omega)=0$ for any $\Omega \subset B(x, 3 R)$. Hence, $\left(\theta_{1}, \ldots, \theta_{n-2}, \theta_{n-1}\right) \in[0, \pi] \times \cdots \times[0, \pi] \times[0,2 \pi]$, and each $\theta_{i}$ is single-valued. Thus,

$$
\begin{aligned}
& \nabla w=-\sin \theta_{1} \nabla \theta_{1} e_{1}+\left(\cos \theta_{1} \cos \theta_{2} \nabla \theta_{1}-\sin \theta_{1} \sin \theta_{2} \nabla \theta_{2}\right) e_{2} \\
& +\left(\cos \theta_{1} \sin \theta_{2} \cos \theta_{3} \nabla \theta_{1}+\sin \theta_{1} \cos \theta_{2} \cos \theta_{3} \nabla \theta_{2}-\sin \theta_{1} \sin \theta_{2} \sin \theta_{3} \nabla \theta_{3}\right) e_{3} \\
& +\cdots+\left(\cos \theta_{1} \sin \theta_{2} \cdots \sin \theta_{n-2} \cos \theta_{n-1} \nabla \theta_{1}+\cdots\right. \\
& \left.\quad+\sin \theta_{1} \cdots \sin \theta_{n-3} \cos \theta_{n-2} \cos \theta_{n-1} \nabla \theta_{n-2}-\sin \theta_{1} \cdots \sin \theta_{n-1} \nabla \theta_{n-1}\right) e_{n-1} \\
& \quad+\left(\cos \theta_{1} \sin \theta_{2} \cdots \sin \theta_{n-1} \nabla \theta_{1}+\cdots+\sin \theta_{1} \cdots \sin \theta_{n-2} \cos \theta_{n-1} \nabla \theta_{n-1}\right) e_{n} .
\end{aligned}
$$

Hence,

(6-2) $|\nabla w|^{2}=\left|\nabla \theta_{1}\right|^{2}+\sin ^{2} \theta_{1}\left|\nabla \theta_{2}\right|^{2}+\sin ^{2} \theta_{1} \sin ^{2} \theta_{2}\left|\nabla \theta_{3}\right|^{2}+\cdots$

$$
+\sin ^{2} \theta_{1} \cdots \sin ^{2} \theta_{n-2}\left|\nabla \theta_{n-1}\right|^{2},
$$


and there are $n(n-1) / 2$ vectors in the formula $w \wedge \nabla w=\left(\left(\cos \theta_{2} \nabla \theta_{1}-\cos \theta_{1} \sin \theta_{1} \sin \theta_{2} \nabla \theta_{2}\right)\left(e_{1} \wedge e_{2}\right)+\cdots\right.$

$$
\begin{array}{r}
+\left(\prod_{i=1}^{n-1} \sin \theta_{i} \nabla \theta_{1}+\cos \theta_{1} \prod_{i=1}^{n-1} \sin \theta_{i} \nabla \theta_{2}+\cdots\right. \\
\left.\left.\quad+\cos \theta_{1} \prod_{i=1}^{n-2} \sin \theta_{i} \cos \theta_{n-1} \nabla \theta_{n-1}\right)\left(e_{1} \wedge e_{n}\right)\right)
\end{array}
$$

$+\left(\left(\sin ^{2} \theta_{1} \cos \theta_{3} \nabla \theta_{2}-\sin ^{2} \theta_{1} \sin \theta_{2} \cos \theta_{2} \sin \theta_{3} \nabla \theta_{3}\right)\left(e_{2} \wedge e_{3}\right)+\cdots\right.$

$$
\begin{aligned}
+ & \left(\sin ^{2} \theta_{1} \prod_{i=3}^{n-1} \sin \theta_{i} \nabla \theta_{2}+\cdots\right. \\
& \left.\left.+\sin ^{2} \theta_{1} \cos \theta_{2} \prod_{i=2}^{n-2} \sin \theta_{i} \cos \theta_{n-1} \nabla \theta_{n-1}\right)\left(e_{2} \wedge e_{n}\right)\right)+\cdots
\end{aligned}
$$

$+\left(\prod_{i=1}^{n-3} \sin ^{2} \theta_{i}\left(\cos \theta_{n-1} \nabla \theta_{n-2}-\sin \theta_{n-2} \cos \theta_{n-2} \sin \theta_{n-1} \nabla \theta_{n-1}\right)\left(e_{n-2} \wedge e_{n-1}\right)\right.$

$\left.+\prod_{i=1}^{n-3} \sin ^{2} \theta_{i}\left(\sin \theta_{n-1} \nabla \theta_{n-2}+\sin \theta_{n-2} \cos \theta_{n-2} \cos \theta_{n-1} \nabla \theta_{n-1}\right)\left(e_{n-2} \wedge e_{n}\right)\right)$

$+\sin ^{2} \theta_{1} \cdots \sin ^{2} \theta_{n-2} \nabla \theta_{n-1}\left(e_{n-1} \wedge e_{n}\right)$.

The equality corresponding to $e_{n-1} \wedge e_{n}$ in the integral system (6-1) is

$$
\int_{B(x, 3 R)}|\nabla u|^{p-2} h^{2} \Pi_{i=1}^{n-2} \sin ^{2} \theta_{i} \nabla \theta_{n-1} \nabla \zeta=0 .
$$

Letting $\zeta=\theta_{n-1} \xi^{2}$ where $\xi \in W_{0}^{1, p}(B(x, 3 R))$, we obtain

$$
\begin{aligned}
\int_{B(x, 3 R)}|\nabla u|^{p-2} h^{2} \prod_{i=1}^{n-2} \sin ^{2} \theta_{i} \mid & \left.\nabla \theta_{n-1}\right|^{2} \xi^{2} \\
\leq & \left.\left|\int_{B(x, 3 R)}\right| \nabla u\right|^{p-2} h^{2} \prod_{i=1}^{n-2} \sin ^{2} \theta_{i}\left(\xi \theta_{n-1}\right) \nabla \theta_{n-1} \nabla \xi \mid .
\end{aligned}
$$

Using Hölder's inequality, we have, for any $\delta \in(0,1)$,

$$
\begin{aligned}
\int_{B(x, 3 R)}|\nabla u|^{p-2} h^{2} & \prod_{i=1}^{n-2} \sin ^{2} \theta_{i}\left|\nabla \theta_{n-1}\right|^{2} \xi^{2} \\
\leq & \delta \int_{B(x, 3 R)}|\nabla u|^{p-2} h^{2} \prod_{i=1}^{n-2} \sin ^{2} \theta_{i}\left|\nabla \theta_{n-1}\right|^{2} \xi^{2} \\
& +C(\delta) \int_{B(x, 3 R)}|\nabla u|^{p-2} h^{2} \prod_{i=1}^{n-2} \sin ^{2} \theta_{i}|\nabla \xi|^{2}\left(\xi \theta_{n-1}\right)^{2} .
\end{aligned}
$$

Taking $\xi=1$ over $B(x, \rho)$ and $\xi=0$ over $B(x, 3 R) \backslash B(x, 2 \rho)$ and letting $\delta$ be sufficiently small, we get

$$
\begin{aligned}
\int_{B(x, \rho)}|\nabla u|^{p-2} h^{2} \prod_{i=1}^{n-2} \sin ^{2} \theta_{i}\left|\nabla \theta_{n-1}\right|^{2} & \leq C \int_{B(x, 2 \rho)}|\nabla u|^{p-2} \\
& \leq C\left(\int_{B(x, 2 \rho)}|\nabla u|^{p}\right)^{1-2 / p} .
\end{aligned}
$$


Next, we use the equalities corresponding to $e_{n-2} \wedge e_{n-1}$ and $e_{n-2} \wedge e_{n}$ in (6-1): the integrals over $B(x, 3 R)$ of

$$
|\nabla u|^{p-2} h^{2} \prod_{i=1}^{n-3} \sin ^{2} \theta_{i}\left(\cos \theta_{n-1} \nabla \theta_{n-2}-\cos \theta_{n-2} \sin \theta_{n-2} \sin \theta_{n-1} \nabla \theta_{n-1}\right) \nabla \zeta
$$

and

$$
|\nabla u|^{p-2} h^{2} \prod_{i=1}^{n-3} \sin ^{2} \theta_{i}\left(\sin \theta_{n-1} \nabla \theta_{n-2}+\cos \theta_{n-2} \sin \theta_{n-2} \cos \theta_{n-1} \nabla \theta_{n-1}\right) \nabla \zeta
$$

both equal zero. Taking $\zeta=\theta_{n-2} \xi^{2} \cos \theta_{n-1}$ and $\theta_{n-2} \xi^{2} \sin \theta_{n-1}$ in these two integrals, respectively, we obtain

$$
\int_{B(x, 3 R)}|\nabla u|^{p-2} h^{2} \prod_{i=1}^{n-3} \sin ^{2} \theta_{i}\left(\left|\nabla \theta_{n-2}\right|^{2} \xi^{2}+\theta_{n-2} \nabla \theta_{n-2} \nabla \xi^{2}\right)=0 .
$$

Similar to the derivation of (6-3), we have, for any $\rho \in(0,3 R / 2)$,

$$
\int_{B(x, \rho)}|\nabla u|^{p-2} h^{2} \prod_{i=1}^{n-3} \sin ^{2} \theta_{i}\left|\nabla \theta_{n-2}\right|^{2} \leq C\left(\int_{B(x, 2 \rho)}|\nabla u|^{p}\right)^{1-2 / p} .
$$

By means of induction, applying the equalities corresponding to $e_{k} \wedge e_{k+1}, e_{k} \wedge e_{k+2}$, $\ldots, e_{k} \wedge e_{n}$ in (6-1), we find that

$$
\int_{B(x, \rho)}|\nabla u|^{p-2} h^{2} \prod_{i=1}^{k-1} \sin ^{2} \theta_{i}\left|\nabla \theta_{k}\right|^{2} \leq C\left(\int_{B(x, 2 \rho)}|\nabla u|^{p}\right)^{1-2 / p},
$$

for $k=2, \ldots, n-1$. At last, we can deduce

$$
\int_{B(x, \rho)}|\nabla u|^{p-2} h^{2}\left|\nabla \theta_{1}\right|^{2} \leq C\left(\int_{B(x, 2 \rho)}|\nabla u|^{p}\right)^{1-2 / p} .
$$

Combining the estimations (6-3)-(6-6) and using (6-2) completes the proof.

\section{References}

[Bethuel et al. 1994] F. Bethuel, H. Brezis, and F. Hélein, Ginzburg-Landau vortices, Progress in Nonlinear Differential Eq. and their Applications, 13, Birkhäuser, Boston, 1994. MR 95c:58044 Zbl 0802.35142

[Chapman et al. 1995] S. J. Chapman, Q. Du, and M. D. Gunzburger, "A Ginzburg-Landau type model of superconducting/normal junctions including Josephson junctions", European J. Appl. Math. 6:2 (1995), 97-114. MR 1331493 (96c:82069) Zbl 0843.35120

[Chen and DiBenedetto 1989] Y. Z. Chen and E. DiBenedetto, "Boundary estimates for solutions of nonlinear degenerate parabolic systems”, J. Reine Angew. Math. 395 (1989), 102-131. MR 983061 (90g:35085) Zbl 0661.35052

[Ding et al. 1998] S. Ding, Z. Liu, and W. Yu, "A variational problem related to the GinzburgLandau model of superconductivity with normal impurity inclusion”, SIAM J. Math. Anal. 29:1 (1998), 48-68. MR 99f:35186 Zbl 0913.35040 
[Du et al. 1992] Q. Du, M. D. Gunzburger, and J. S. Peterson, "Analysis and approximation of the Ginzburg-Landau model of superconductivity", SIAM Rev. 34:1 (1992), 54-81. MR 93g:82109 Zbl 0787.65091

[Han and Li 1996] Z.-C. Han and Y. Y. Li, "Degenerate elliptic systems and applications to GinzburgLandau type equations. I", Calc. Var. Partial Differential Eq. 4:2 (1996), 171-202. MR 97d:35085a Zbl 0847.35055

[Hong 1996] M.-C. Hong, "Asymptotic behavior for minimizers of a Ginzburg-Landau-type functional in higher dimensions associated with $n$-harmonic maps", Adv. Differential Equations 1:4 (1996), 611-634. MR 97h:58047 Zbl 0857.35120

[Lei 2004] Y. Lei, " $C^{1, \alpha}$ convergence of a Ginzburg-Landau type minimizer in higher dimensions", Nonlinear Anal. 59:4 (2004), 609-627. MR 2094431 (2005h:35129) Zbl pre02117582

[Lei and $\mathrm{Wu} 2000$ ] Y. Lei and $\mathrm{Z}$. Wu, " $C^{1, \alpha}$ convergence of minimizers of a Ginzburg-Landau functional”, Electron. J. Differential Equations 14 (2000), 20. MR 2001a:58012 Zbl 0939.35076

[Misawa 2001] M. Misawa, "Approximation of $p$-harmonic maps by the penalized equation", Nonlinear Anal. 47:2 (2001), 1069-1080. MR 2003m:58021 Zbl 1042.58507

[Struwe 1993] M. Struwe, "Une estimation asymptotique pour le modèle de Ginzburg-Landau", $C$. R. Acad. Sci. Paris Sér. I Math. 317:7 (1993), 677-680. MR 94k:35043 Zbl 0789.49005

[Tinkham 1975] M. Tinkham, Introduction to superconductivity, McGraw-Hill, New York, 1975.

[Tolksdorf 1983] P. Tolksdorf, "Everywhere-regularity for some quasilinear systems with a lack of ellipticity”, Ann. Mat. Pura Appl. (4) 134 (1983), 241-266. MR 85h:35104 Zbl 0538.35034

Received October 9, 2004.

\section{YUTIAN LEI}

DEPARTMENT OF MATHEMATICS

NANJING NORMAL UNIVERSITY,

NANJING, JIANGSU, 210097 ,

P.R.CHINA

leiyutian@njnu.edu.cn 